\title{
Filtering for Uncertain Two-dimensional Discrete Systems with State Delays
}

\author{
Ligang Wu, Zidong Wang*, Huijun Gao and Changhong Wang
}

\begin{abstract}
This paper is concerned with the problem of robust $\mathcal{H}_{\infty}$ filtering for two-dimensional (2-D) discrete systems with time delays in states. The 2-D systems under consideration are described in terms of the well-known Fornasini-Marchesini local state-space (FMLSS) models with time-delays. Our attention is focused on the design of a full-order filter such that the filtering error system is guaranteed to be asymptotically stable with a prescribed $\mathcal{H}_{\infty}$ disturbance attenuation performance. Sufficient conditions for the existence of desired filters are established by using a linear matrix inequality (LMI) approach, and the corresponding filter design problem is then cast into a convex optimization problem that can be efficiently solved by resorting to some standard numerical software. Furthermore, the obtained results are extended to more general cases where the system matrices contain either polytopic or norm-bounded parameter uncertainties. A simulation example is provided to illustrate the effectiveness of the proposed design method.
\end{abstract}

\section{Keywords}

Filtering, $\mathcal{H}_{\infty}$ norm, linear matrix inequality (LMI), time-delay, two-dimensional (2-D) systems

\section{INTRODUCTION}

In the past few decades, two-dimensional (2-D) discrete-time systems have received considerable research attention since 2-D systems have extensive applications in image processing, seismographic data processing, thermal processes, water stream heating, etc., see [13]. So far, many important results have been reported in the literature. For example, the stability analysis problem for 2-D systems has been investigated in $[8,15]$, the controller and filter design problems have been considered in [4,5, 19,26], and the model approximation problem for 2-D digital filters has been studied in [7].

On the other hand, in the signal processing and control communities, the $\mathcal{H}_{\infty}$ filtering problem has recently drawn a great deal of research interests. The aim of $\mathcal{H}_{\infty}$ filtering problem is basically to find a full-order (or reduced-order) filter such that the associated filtering error system satisfies a prescribed $\mathcal{H}_{\infty}$ norm bound constraint. Much work has been done for $\mathcal{H}_{\infty}$ filtering problem, see e.g. [1,3,10,11,22-24] and references therein. It has also been well recognized that time delay exists commonly in dynamic systems and is frequently a source of instability and poor performance. Therefore, the last ten years have witnessed significant advances in dealing with analysis and design problems for time-delay systems. In particular, the $\mathcal{H}_{\infty}$ filtering problem has been thoroughly studied for various time-delay systems, see e.g. [9,14,18,25,27-29] for some recent papers. However, the aforementioned results are only concerned with one-dimensional (1-D) time-delay systems. When it comes to the 2-D systems, most published results have been restricted to the 2-D discrete-time delay free systems, see $[4,12,20]$. In the simultaneous presence of time-delays and parameter uncertainties, unfortunately, the robust $\mathcal{H}_{\infty}$ filtering problem for 2-D discrete-time systems has not gained enough research attention mainly due to the complexity in the stability analysis, despite its potential in engineering applications. This situation motivates our current investigation.

L. Wu, H. Gao and C. Wang are with the Space Control and Inertial Technology Research Center, Harbin Institute of Technology, Harbin, 150001, P. R. China. Email: \{ligangwu; hjgao; cwang\}@hit.edu.cn

Z. Wang is with the Department of Information Systems and Computing, Brunel University, Uxbridge, Middlesex, UB8 3PH, United Kingdom.

${ }^{*}$ Corresponding author. Email: Zidong. Wang@brunel.ac.uk 
It is, therefore, our intention in this paper to investigate the problem of $\mathcal{H}_{\infty}$ filtering for 2-D systems with time-delay in states. The mathematical model of the 2-D systems is established in terms of the well-known Fornasini-Marchesini local state-space (FMLSS) model incorporating time delays. We aim at designing a fullorder filter that guarantees the asymptotic stability of the filtering error system while keeping the prescribed $\mathcal{H}_{\infty}$ disturbance attenuation performance. By using a linear matrix inequality (LMI) approach, we derive the existence conditions of the desired filters, and convert the corresponding filter design problem into a convex optimization one that can then be efficiently handled with help from available numerical software [2]. Furthermore, the obtained results are extended to some more general cases where the system matrices also contain uncertain parameters. Most frequently used descriptions for the parameter uncertainties, including polytopic and norm-bounded characterizations, are taken into consideration within the unified LMI framework. A numerical example is provided to demonstrate the effectiveness of the proposed filter design procedures.

The remainder of this paper is organized as follows. The problems of $\mathcal{H}_{\infty}$ filtering for 2-D discrete statedelayed systems is formulated in Section II. Section III presents our main results of filtering for 2-D discretetime systems with state delays, and the results obtained are further extended in Section IV to more general cases where the parameter uncertainties are considered. Section V provides an illustrative example and we conclude this paper in Section VI.

Notations. The notations used throughout the paper are fairly standard. The superscript " $T$ " stands for matrix transposition; $\mathbb{R}^{n}$ denotes the $n$-dimensional Euclidean space; $\mathbb{R}^{m \times n}$ is the set of all real matrices of dimension $m \times n$ and the notation $P>0$ means that $P$ is real symmetric and positive definite; $I$ and 0 represent identity matrix and zero matrix; $|\cdot|$ refers to the Euclidean vector norm; and $\lambda_{\min }(\cdot), \lambda_{\max }(\cdot)$ denote the minimum and the maximum eigenvalues of a real symmetric matrix, respectively. In symmetric block matrices or long matrix expressions, we use an asterisk $(*)$ to represent a term that is induced by symmetry, and $\operatorname{diag}\{\ldots\}$ stands for a block-diagonal matrix. $l_{2}^{n}\{[0, \infty),[0, \infty)\}$ denotes the space of square summable sequences on $\{[0, \infty),[0, \infty)\}$ with values on $\mathbb{R}^{n}$. Matrices, if their dimensions are not explicitly stated, are assumed to be compatible for algebraic operations.

\section{Problem Formulation}

Consider the following state delayed 2-D system $\Sigma_{F M}$ described by the FMLSS model [6] with delays in the states:

$$
\begin{aligned}
\Sigma_{F M}: \quad x(i+1, j+1)= & A_{1} x(i, j+1)+A_{2} x(i+1, j)+A_{d 1} x\left(i-d_{1}, j+1\right)+A_{d 2} x\left(i+1, j-d_{2}\right) \\
& +B_{1} \omega(i, j+1)+B_{2} \omega(i+1, j) \\
y(i, j)= & C x(i, j)+D \omega(i, j) \\
z(i, j)= & E x(i, j)
\end{aligned}
$$

where $x(i, j) \in \mathbb{R}^{n}$ is the state; $\omega(i, j) \in l_{2}^{l}\{[0, \infty),[0, \infty)\}$ is the disturbance input; $y(i, j) \in \mathbb{R}^{m}$ is the measured output; $z(i, j) \in \mathbb{R}^{p}$ is the signal to be estimated with $i, j \in \mathbb{Z}_{+}$; and $d_{1}$ and $d_{2}$ are constant positive integers representing delays along vertical and horizontal directions, respectively. $A_{1}, A_{2}, A_{d 1}, A_{d 2}, B_{1}, B_{2}$, $C, D$ and $E$ are constant matrices with compatible dimensions. The boundary conditions are given by

$$
\begin{aligned}
& \{x(\phi, j)=0\}, \quad \forall j \geq 0, \phi=-d_{1},-d_{1}+1, \ldots, 0 \\
& \{x(i, \varphi)=0\}, \quad \forall i \geq 0, \varphi=-d_{2},-d_{2}+1, \ldots, 0
\end{aligned}
$$

Throughout this paper, the following assumptions are made.

Assumption 1: System $\Sigma_{F M}$ in (1) is asymptotically stable. 
Assumption 2: The boundary condition is assumed to satisfy

$$
\lim _{N \rightarrow \infty} \sum_{k=0}^{N}\left(\left|x_{0, k}\right|^{2}+\left|x_{k, 0}\right|^{2}\right)<\infty
$$

The aim of the robust $\mathcal{H}_{\infty}$ filtering problem addressed in this paper is to estimate the signal $z(i, j)$ by a linear, full-order, dynamic filter of the structure described by $\hat{\Sigma}_{F M}$ :

$$
\begin{aligned}
\hat{\Sigma}_{F M}: \quad x_{f}(i+1, j+1) & =A_{1 f} x_{f}(i, j+1)+A_{2 f} x_{f}(i+1, j)+B_{1 f} y(i, j+1)+B_{2 f} y(i+1, j) \\
z_{f}(i, j) & =C_{f} x_{f}(i, j) \\
x_{f}(i, j) & =0 \quad \text { for } i=0 \text { or } j=0
\end{aligned}
$$

where $x_{f}(i, j) \in \mathbb{R}^{n}$ is the filter state vector, and $A_{1 f}, A_{2 f}, B_{1 f}, B_{2 f}$ and $C_{f}$ are appropriately dimensioned constant matrices to be determined.

Now, augmenting the model of $\Sigma_{F M}$ to include the states of filter $\hat{\Sigma}_{F M}$, we obtain the following filtering error system $\tilde{\Sigma}_{F M}$ :

$$
\begin{aligned}
\tilde{\Sigma}_{F M}: \quad \xi(i+1, j+1)= & \tilde{A}_{1} \xi(i, j+1)+\tilde{A}_{2} \xi(i+1, j)+\tilde{A}_{d 1} \xi\left(i-d_{1}, j+1\right)+\tilde{A}_{d 2} \xi\left(i+1, j-d_{2}\right) \\
& +\tilde{B}_{1} \omega(i, j+1)+\tilde{B}_{2} \omega(i+1, j) \\
e(i, j)= & \tilde{C} \xi(i, j)
\end{aligned}
$$

where $\xi(i, j) \triangleq\left[x^{T}(i, j) \quad x_{f}^{T}(i, j)\right]^{T}, e(i, j) \triangleq z(i, j)-z_{f}(i, j)$ and

$$
\begin{aligned}
& \tilde{A}_{1} \triangleq\left[\begin{array}{cc}
A_{1} & 0 \\
B_{1 f} C & A_{1 f}
\end{array}\right], \quad \tilde{A}_{2} \triangleq\left[\begin{array}{cc}
A_{2} & 0 \\
B_{2 f} C & A_{2 f}
\end{array}\right], \quad \tilde{A}_{d 1} \triangleq\left[\begin{array}{cc}
A_{d 1} & 0 \\
0 & 0
\end{array}\right], \\
& \tilde{A}_{d 2} \triangleq\left[\begin{array}{cc}
A_{d 2} & 0 \\
0 & 0
\end{array}\right], \quad \tilde{B}_{1} \triangleq\left[\begin{array}{c}
B_{1} \\
B_{1 f} D
\end{array}\right], \quad \tilde{B}_{2} \triangleq\left[\begin{array}{c}
B_{2} \\
B_{2 f} D
\end{array}\right], \quad \tilde{C} \triangleq\left[\begin{array}{ll}
E & -C_{f}
\end{array}\right]
\end{aligned}
$$

Before problem formulating, we give the following definitions.

Definition 1: Consider the filtering error system $\tilde{\Sigma}_{F M}$ in (5). Given a scalar $\gamma>0$ and constant weighting matrices $\tilde{P}>0, \tilde{Q}>0, \tilde{Q}_{1}>0$ and $\tilde{Q}_{2}>0$, the system $\tilde{\Sigma}_{F M}$ is said to have an $\mathcal{H}_{\infty}$ performance level $\gamma$ if it is asymptotically stable and satisfies

$$
\begin{gathered}
\frac{\|\bar{e}(i, j)\|_{2}^{2}}{\left[\|\bar{\omega}(i, j)\|_{2}^{2}+\sum_{j=0}^{\infty} \xi_{01}^{T}(0, j) \tilde{P} \xi_{01}(0, j)+\sum_{i=0}^{\infty} \xi_{10}^{T}(i, 0) \tilde{Q} \xi_{10}(i, 0)\right.}<\gamma^{2} \\
\left.+\sum_{j=0}^{\infty} \sum_{\kappa=-d_{1}}^{-1} \xi_{\kappa 1}^{T}(0, j) \tilde{Q}_{1} \xi_{\kappa 1}(0, j)+\sum_{i=0}^{\infty} \sum_{\kappa=-d_{2}}^{-1} \xi_{1 \kappa}^{T}(i, 0) \tilde{Q}_{2} \xi_{1 \kappa}(i, 0)\right]
\end{gathered}
$$

where $\xi_{\alpha \beta}(i, j) \triangleq \xi(i+\alpha, j+\beta)$. In the case of the zero boundary conditions as in $(2)$, the above $\mathcal{H}_{\infty}$ performance measure (7) reduces to

$$
\|\bar{e}(i, j)\|_{2}<\gamma\|\bar{\omega}(i, j)\|_{2} \quad(\gamma>0)
$$

where $\bar{e}(i, j) \triangleq\left[\begin{array}{lll}e^{T}(i, j+1) & e^{T}(i+1, j)\end{array}\right]^{T}, \bar{\omega}(i, j) \triangleq\left[\begin{array}{ll}\omega^{T}(i, j+1) & \omega^{T}(i+1, j)\end{array}\right]^{T}$ and $\|\cdot\|_{2}$ is $l_{2}$ norm defined by

$$
\begin{aligned}
\|\bar{e}(i, j)\|_{2}^{2} & \triangleq \sum_{i=0}^{\infty} \sum_{j=0}^{\infty}\left[e^{T}(i, j+1) e(i, j+1)+e^{T}(i+1, j) e(i+1, j)\right] \\
\|\bar{\omega}(i, j)\|_{2}^{2} & \triangleq \sum_{i=0}^{\infty} \sum_{j=0}^{\infty}\left[\omega^{T}(i, j+1) \omega(i, j+1)+\omega^{T}(i+1, j) \omega(i+1, j)\right]
\end{aligned}
$$


Definition 2: The filter $\hat{\Sigma}_{F M}$ in (4) is said to be an $\mathcal{H}_{\infty}$ filter if the filtering error system $\tilde{\Sigma}_{F M}$ in (5) is asymptotically stable and satisfies $\mathcal{H}_{\infty}$ performance in (8) with zero boundary conditions as in (2).

The objective of this paper is to find the matrices $A_{1 f}, A_{2 f}, B_{1 f}, B_{2 f}$ and $C_{f}$ of the full-order $\mathcal{H}_{\infty}$ filter $\hat{\Sigma}_{F M}$ in (4) for the 2-D state-delayed system $\Sigma_{F M}$ in (1), such that for any nonzero $\omega(i, j) \in l_{2}\{[0, \infty),[0, \infty)\}$ the filtering error system $\tilde{\Sigma}_{F M}$ in (5) is asymptotically stable and satisfies (8).

\section{MAIN ReSUlts}

\section{A. Filter Analysis}

In this subsection, we shall analyze the stability and $\mathcal{H}_{\infty}$ performance for the filtering error system $\tilde{\Sigma}_{F M}$.

The following lemma is essential in establishing our stability results.

Lemma 1: (Theorem 3 of [19]) The 2D state-delayed system $\tilde{\Sigma}_{F M}$ in (5) with $\omega_{i, j} \equiv 0$ is asymptotically stable if there exist matrices $P>0, Q>0, Q_{1}>0$ and $Q_{2}>0$ such that the following LMI holds:

$$
\left[\begin{array}{c}
\tilde{A}_{1}^{T} \\
\tilde{A}_{2}^{T} \\
\tilde{A}_{d 1}^{T} \\
\tilde{A}_{d 2}^{T}
\end{array}\right] P\left[\begin{array}{llll}
\tilde{A}_{1} & \tilde{A}_{2} & \tilde{A}_{d 1} & \tilde{A}_{d 2}
\end{array}\right]-\left[\begin{array}{cccc}
P-Q-Q_{1} & 0 & 0 & 0 \\
* & Q-Q_{2} & 0 & 0 \\
* & * & Q_{1} & 0 \\
* & * & * & Q_{2}
\end{array}\right]<0 .
$$

Next, the following Theorem provides a sufficient condition under which the filtering error system $\tilde{\Sigma}_{F M}$ in (5) is asymptotically stable and the performance constraint (8) is satisfied.

Theorem 1: The filtering error system $\tilde{\Sigma}_{F M}$ in (5) is asymptotically stable with an $\mathcal{H}_{\infty}$ disturbance attenuation level bound $\gamma$ if there exist matrices $P>0, Q>0, Q_{1}>0$ and $Q_{2}>0$ such that the following LMI holds:

$$
\left[\begin{array}{ccccccccc}
-P & 0 & 0 & P \tilde{A}_{1} & P \tilde{B}_{1} & P \tilde{A}_{d 1} & P \tilde{A}_{2} & P \tilde{B}_{2} & P \tilde{A}_{d 2} \\
* & -I & 0 & \tilde{C} & 0 & 0 & 0 & 0 & 0 \\
* & * & -I & 0 & 0 & 0 & \tilde{C} & 0 & 0 \\
* & * & * & Q+Q_{1}-P & 0 & 0 & 0 & 0 & 0 \\
* & * & * & * & -\gamma^{2} I & 0 & 0 & 0 & 0 \\
* & * & * & * & * & -Q_{1} & 0 & 0 & 0 \\
* & * & * & * & * & * & Q_{2}-Q & 0 & 0 \\
* & * & * & * & * & * & * & -\gamma^{2} I & 0 \\
* & * & * & * & * & * & * & * & -Q_{2}
\end{array}\right]<0 .
$$

Proof. See the Appendix.

For the delay free case, i.e., $\tilde{A}_{d 1}=0$ and $\tilde{A}_{d 2}=0$, according to the procedure of the proof of Theorem 1 , it is clear that setting $Q_{1}=0$ and $Q_{2}=0$ in Theorem 1 would yield the following Corollary.

Corollary 1: The filtering error system $\tilde{\Sigma}_{F M}$ in (5) is asymptotically stable with an $\mathcal{H}_{\infty}$ disturbance attenuation level bound $\gamma$ if there exist matrices $P>0$ and $Q>0$ such that the following LMI holds:

$$
\left[\begin{array}{ccccccc}
-P & 0 & 0 & P \bar{A}_{1} & P \bar{B}_{1} & P \bar{A}_{2} & P \bar{B}_{2} \\
* & -I & 0 & \bar{C} & 0 & 0 & 0 \\
* & * & -I & 0 & 0 & \bar{C} & 0 \\
* & * & * & Q-P & 0 & 0 & 0 \\
* & * & * & * & -\gamma^{2} I & 0 & 0 \\
* & * & * & * & * & -Q & 0 \\
* & * & * & * & * & * & -\gamma^{2} I
\end{array}\right]<0
$$

Remark 1: It should be pointed out that the result in Corollary 1 is actually the main result in [7]. In other words, Theorem 1 in this paper is an extension of the main result of [7]. 


\section{B. Filter Synthesis}

We are now ready to deal with the $\mathcal{H}_{\infty}$ filter design problem in the following theorem.

Theorem 2: Consider the 2-D state-delayed system $\Sigma_{F M}$ in (1) and let $\gamma>0$ be a prescribed constant scalar. Then there exists a full-order filter $\hat{\Sigma}_{F M}$ in the form of (4) such that the filtering error system $\tilde{\Sigma}_{F M}$ is asymptotically stable and (8) is satisfied if there exist matrices $\mathcal{U}>0, \mathcal{V}>0, \mathcal{Q}_{1}>0, \mathcal{Q}_{3}>0, \mathcal{Q}_{11}>0$, $\mathcal{Q}_{13}>0, \mathcal{Q}_{21}>0, \mathcal{Q}_{23}>0, \mathcal{Q}_{2}, \mathcal{Q}_{12}, \mathcal{Q}_{22}, \mathcal{A}_{1 f}, \mathcal{A}_{2 f}, \mathcal{B}_{1 f}, \mathcal{B}_{2 f}$ and $\mathcal{C}_{f}$ such that the following LMIs hold:

$$
\begin{aligned}
& {\left[\begin{array}{cccccccc}
-\mathcal{U} & -\mathcal{V} & 0 & 0 & \mathcal{U} A_{1}+\mathcal{B}_{1 f} C & \mathcal{A}_{1 f} & \mathcal{U} B_{1}+\mathcal{B}_{1 f} D & \mathcal{U} A_{d 1} \\
* & -\mathcal{V} & 0 & 0 & \mathcal{V} A_{1}+\mathcal{B}_{1 f} C & \mathcal{A}_{1 f} & \mathcal{V} B_{1}+\mathcal{B}_{1 f} D & \mathcal{V} A_{d 1} \\
* & * & -I & 0 & E & -\mathcal{C}_{f} & 0 & 0 \\
* & * & * & -I & 0 & 0 & 0 & 0 \\
* & * & * & * & \mathcal{Q}_{1}+\mathcal{Q}_{11}-\mathcal{U} & \mathcal{Q}_{2}+\mathcal{Q}_{12}-\mathcal{V} & 0 & 0 \\
* & * & * & * & * & \mathcal{Q}_{3}+\mathcal{Q}_{13}-\mathcal{V} & 0 & 0 \\
* & * & * & * & * & * & -\gamma^{2} I & 0 \\
* & * & * & * & * & * & * & -\mathcal{Q}_{11} \\
* & * & * & * & * & * & * & * \\
* & * & * & * & * & * & * & * \\
* & * & * & * & * & * & * & * \\
* & * & * & * & * & * & * & * \\
* & * & * & * & * & * & * & * \\
* & * & * & * & * & * & * & *
\end{array}\right]} \\
& \begin{array}{llllll}
0 & \mathcal{U} A_{2}+\mathcal{B}_{2 f} C & \mathcal{A}_{2 f} & \mathcal{U} B_{2}+\mathcal{B}_{2 f} D & \mathcal{U} A_{d 2} & 0
\end{array} \\
& \begin{array}{llllll}
0 & \mathcal{V} A_{2}+\mathcal{B}_{2 f} C & \mathcal{A}_{2 f} & \mathcal{V} B_{2}+\mathcal{B}_{2 f} D & \mathcal{V} A_{d 2} & 0
\end{array} \\
& \begin{array}{llllll}
0 & 0 & 0 & 0 & 0 & 0
\end{array} \\
& \begin{array}{llllll}
0 & E & -\mathcal{C}_{f} & 0 & 0 & 0
\end{array} \\
& \begin{array}{llllll}
0 & 0 & 0 & 0 & 0 & 0
\end{array} \\
& \begin{array}{llllll}
0 & 0 & 0 & 0 & 0 & 0
\end{array} \\
& \begin{array}{cccccc}
0 & 0 & 0 & 0 & 0 & 0 \\
-\mathcal{Q}_{12} & 0 & 0 & 0 & 0 & 0
\end{array}<0 \text {, } \\
& \begin{array}{llllll}
-\mathcal{Q}_{13} & 0 & 0 & 0 & 0 & 0
\end{array} \\
& \text { * } \quad \mathcal{Q}_{21}-\mathcal{Q}_{1} \quad \mathcal{Q}_{22}-\mathcal{Q}_{2} \quad 0 \quad 000 \\
& \begin{array}{llllll}
* & * & \mathcal{Q}_{23}-\mathcal{Q}_{3} & 0 & 0 & 0
\end{array}
\end{aligned}
$$

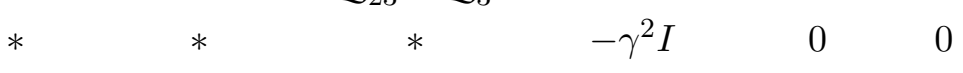

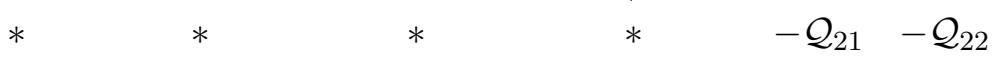

$$
\begin{aligned}
& * \quad * \quad * \quad * \quad \begin{array}{lllll}
* & * & & * & -\mathcal{Q}_{23}
\end{array} \\
& {\left[\begin{array}{cc}
\mathcal{Q}_{1} & \mathcal{Q}_{2} \\
* & \mathcal{Q}_{3}
\end{array}\right]>0,} \\
& {\left[\begin{array}{cc}
\mathcal{Q}_{11} & \mathcal{Q}_{12} \\
* & \mathcal{Q}_{13}
\end{array}\right]>0,} \\
& {\left[\begin{array}{cc}
\mathcal{Q}_{21} & \mathcal{Q}_{22} \\
* & \mathcal{Q}_{23}
\end{array}\right]>0 .}
\end{aligned}
$$


Moreover, the parameters of a desired $\mathcal{H}_{\infty}$ filter of the form (4) can be computed from

$$
\left[\begin{array}{cc}
A_{1 f} & B_{1 f} \\
A_{2 f} & B_{2 f} \\
C_{f} & 0
\end{array}\right]=\left[\begin{array}{ccc}
\mathcal{V}^{-1} & 0 & 0 \\
* & \mathcal{V}^{-1} & 0 \\
* & * & I
\end{array}\right]\left[\begin{array}{cc}
\mathcal{A}_{1 f} & \mathcal{B}_{1 f} \\
\mathcal{A}_{2 f} & \mathcal{B}_{2 f} \\
\mathcal{C}_{f} & 0
\end{array}\right]
$$

Proof. According to Theorem 1, if (9) holds $P$ is nonsingular since $P>0$. Now, partition $P$ as

$$
P \triangleq\left[\begin{array}{ll}
P_{1} & P_{2} \\
P_{2}^{T} & P_{3}
\end{array}\right]
$$

Without loss of generality, we assume that $P_{2}$ is nonsingular (if not, $P_{2}$ may be perturbed by a matrix $\Delta P_{2}$ with sufficiently small norm such that $P_{2}+\Delta P_{2}$ is nonsingular and satisfies (9)). Define the following matrices:

$$
\begin{aligned}
& \Gamma \triangleq\left[\begin{array}{cc}
I & 0 \\
0 & P_{3}^{-1} P_{2}^{T}
\end{array}\right], \quad \mathcal{U} \triangleq P_{1}>0, \quad \mathcal{V} \triangleq P_{2} P_{3}^{-1} P_{2}^{T}>0 \\
& \mathbb{Q} \triangleq \Gamma^{T} Q \Gamma \triangleq\left[\begin{array}{cc}
\mathcal{Q}_{1} & \mathcal{Q}_{2} \\
* & \mathcal{Q}_{3}
\end{array}\right]>0, \quad \mathbb{Q}_{1} \triangleq \Gamma^{T} Q_{1} \Gamma \triangleq\left[\begin{array}{cc}
\mathcal{Q}_{11} & \mathcal{Q}_{12} \\
* & \mathcal{Q}_{13}
\end{array}\right]>0 \\
& \mathbb{Q}_{2} \triangleq \Gamma^{T} Q_{2} \Gamma \triangleq\left[\begin{array}{cc}
\mathcal{Q}_{21} & \mathcal{Q}_{22} \\
* & \mathcal{Q}_{23}
\end{array}\right]>0
\end{aligned}
$$

and

$$
\left[\begin{array}{cc}
\mathcal{A}_{1 f} & \mathcal{B}_{1 f} \\
\mathcal{A}_{2 f} & \mathcal{B}_{2 f} \\
\mathcal{C}_{f} & 0
\end{array}\right] \triangleq\left[\begin{array}{ccc}
P_{2} & 0 & 0 \\
* & P_{2} & 0 \\
* & * & I
\end{array}\right]\left[\begin{array}{cc}
A_{1 f} & B_{1 f} \\
A_{2 f} & B_{2 f} \\
C_{f} & 0
\end{array}\right]\left[\begin{array}{cc}
P_{3}^{-1} P_{2}^{T} & 0 \\
0 & I
\end{array}\right]
$$

Performing congruence transformations to (9) by matrix $\operatorname{diag}\{\Gamma, I, I, \Gamma, I, \Gamma, \Gamma, I, \Gamma\}$, we have

$$
\left[\begin{array}{ccccccccc}
-\Gamma^{T} P \Gamma & 0 & 0 & \Gamma^{T} P \tilde{A}_{1} \Gamma & \Gamma^{T} P \tilde{B}_{1} & \Gamma^{T} P \tilde{A}_{d 1} \Gamma & \Gamma^{T} P \tilde{A}_{2} \Gamma & \Gamma^{T} P \tilde{B}_{2} & \Gamma^{T} P \tilde{A}_{d 2} \Gamma \\
* & -I & 0 & \tilde{C} \Gamma & 0 & 0 & 0 & 0 & 0 \\
* & * & -I & 0 & 0 & 0 & \tilde{C} \Gamma & 0 & 0 \\
* & * & * & \mathbb{Q}+\mathbb{Q}_{1}-\Gamma^{T} P \Gamma & 0 & 0 & 0 & 0 & 0 \\
* & * & * & * & -\gamma^{2} I & 0 & 0 & 0 & 0 \\
* & * & * & * & * & -\mathbb{Q}_{1} & 0 & 0 & 0 \\
* & * & * & * & * & * & \mathbb{Q}_{2}-\mathbb{Q} & 0 & 0 \\
* & * & * & * & * & * & * & -\gamma^{2} I & 0 \\
* & * & * & * & * & * & * & * & -\mathbb{Q}_{2}
\end{array}\right]
$$

in which

$$
\begin{aligned}
\Gamma^{T} P^{T} \tilde{A}_{j} \Gamma & =\left[\begin{array}{cc}
P_{1} A_{j}+P_{2} B_{j f} C & P_{2} A_{j f} P_{3}^{-1} P_{2}^{T} \\
P_{2} P_{3}^{-T} P_{2}^{T} A_{j}+P_{2} B_{j f} C & P_{2} A_{j f} P_{3}^{-1} P_{2}^{T}
\end{array}\right], \quad(j=1,2) \\
\Gamma^{T} P^{T} \tilde{A}_{d j} \Gamma & =\left[\begin{array}{cc}
P_{1} A_{d j} & 0 \\
P_{2} P_{3}^{-T} P_{2}^{T} A_{d j} & 0
\end{array}\right], \quad \Gamma^{T} P^{T} \tilde{B}_{j}=\left[\begin{array}{c}
P_{1} B_{j}+P_{2} B_{j f} D \\
P_{2} P_{3}^{-T} P_{2}^{T} B_{j}+P_{2} B_{j f} D
\end{array}\right], \\
\Gamma^{T} P^{T} \Gamma & =\left[\begin{array}{cc}
P_{1} & P_{2} P_{3}^{-1} P_{2}^{T} \\
P_{2} P_{3}^{-T} P_{2}^{T} & P_{2} P_{3}^{-1} P_{2}^{T}
\end{array}\right], \quad \tilde{C} \Gamma=\left[\begin{array}{ll}
E & -C_{f} P_{3}^{-1} P_{2}^{T}
\end{array}\right]
\end{aligned}
$$

Substituting (15)-(17) and (19) into (18), we can obtain (10). Also, from (16), we can obtain (11)-(13). 
On the other hand, (17) is equivalent to

$$
\left[\begin{array}{cc}
A_{1 f} & B_{1 f} \\
A_{2 f} & B_{2 f} \\
C_{f} & 0
\end{array}\right]=\left[\begin{array}{ccc}
P_{2}^{-1} & 0 & 0 \\
* & P_{2}^{-1} & 0 \\
* & * & I
\end{array}\right]\left[\begin{array}{cc}
\mathcal{A}_{1 f} & \mathcal{B}_{1 f} \\
\mathcal{A}_{2 f} & \mathcal{B}_{2 f} \\
\mathcal{C}_{f} & 0
\end{array}\right]\left[\begin{array}{cc}
P_{2}^{-T} P_{3} & 0 \\
0 & I
\end{array}\right]
$$

and it follows from (4) that the transfer function of filter $\hat{\Sigma}_{F M}$ in (4) can be described by

$$
\mathcal{T}\left(z_{1}, z_{2}\right)=C_{f}\left[z_{1} z_{2} I-z_{1} A_{1 f}-z_{2} A_{2 f}\right]^{-1}\left[z_{1} B_{1 f}+z_{2} B_{2 f}\right] .
$$

Substituting (20) into (21) results in

$$
\begin{aligned}
\mathcal{T}\left(z_{1}, z_{2}\right) & =\mathcal{C}_{f} P_{2}^{-T} P_{3}\left[z_{1} z_{2} I-z_{1} P_{2}^{-1} \mathcal{A}_{1 f} P_{2}^{-T} P_{3}-z_{2} P_{2}^{-1} \mathcal{A}_{2 f} P_{2}^{-T} P_{3}\right]^{-1}\left[z_{1} P_{2}^{-1} \mathcal{B}_{1 f}+z_{2} P_{2}^{-1} \mathcal{B}_{2 f}\right] \\
& =\mathcal{C}_{f}\left[z_{1} z_{2} I-z_{1} \mathcal{V}^{-1} \mathcal{A}_{1 f}-z_{2} \mathcal{V}^{-1} \mathcal{A}_{2 f}\right]^{-1}\left[z_{1} \mathcal{V}^{-1} \mathcal{B}_{1 f}+z_{2} \mathcal{V}^{-1} \mathcal{B}_{2 f}\right]
\end{aligned}
$$

Then, the realization of the filter in (14) can be readily established, which completes the proof.

Remark 2: Note that Theorem 2 provides a sufficient condition for the solvability of $\mathcal{H}_{\infty}$ filter design problem for the 2-D state-delayed system. Since the obtained conditions are expressed by strict LMIs, the desired filter can be determined by solving the following convex optimization problem:

$$
\min \delta \quad \text { subject to (10)-(13) with } \delta \triangleq \gamma^{2} \text {. }
$$

\section{Further Extensions}

In this section, we further extend the results obtained so far to 2-D state-delayed systems with uncertain model data, that is, the uncertain parameters are present in the system matrices $A_{1}, A_{2}, A_{d 1}, A_{d 2}, B_{1}, B_{2}$, $C, D$ and $E$. In the following, we will consider two types of parameter uncertainties: polytopic uncertainty and norm-bounded uncertainty, which have been extensively used for studying robust control and filtering problems in the literature (see, for instance, [21] and the references therein).

\section{A. Polytopic Uncertain Case}

Theorem 2 addresses the $\mathcal{H}_{\infty}$ filtering problem for system $\Sigma_{F M}$ in (1) where the system matrices are all known. However, since LMIs (10)-(13) are affine in the system matrices, Theorem 2 can be directly used to solve the $\mathcal{H}_{\infty}$ filtering problem for the case where the system matrices are not exactly known but reside within a given polytope.

Assumption 3: The matrices $A_{1}, A_{2}, A_{d 1}, A_{d 2}, B_{1}, B_{2}, C, D$ and $E$ of system $\Sigma_{F M}$ in (1) contain partially unknown parameters. Assume that $\Omega \triangleq\left(A_{1}, A_{2}, A_{d 1}, A_{d 2}, B_{1}, B_{2}, C, D, E\right) \in \chi$, where $\chi$ is a given convex bounded polyhedral domain described by $s$ vertices:

$$
\chi \triangleq\left\{\chi(\lambda) \mid \chi(\lambda)=\sum_{i=1}^{s} \lambda_{j} \chi_{j} ; \sum_{i=1}^{s} \lambda_{j}=1, \lambda_{j} \geq 0\right\}
$$

where $\chi_{j} \triangleq\left(A_{1 j}, A_{2 j}, A_{d 1 j}, A_{d 2 j}, B_{1 j}, B_{2 j}, C_{j}, D_{j}, E_{j}\right)$ denotes the $j$ th vertex of the polytope $\chi$.

We state the following theorem without proof, since the proof can be obtained along the same line of the derivation of Theorem 2 .

Theorem 3: Consider the 2-D state-delayed system $\Sigma_{F M}$ in (1) with Assumption 3 and let $\gamma>0$ be a prescribed constant scalar. Then there exists a full-order filter $\hat{\Sigma}_{F M}$ in the form of (4) such that the filtering error system $\tilde{\Sigma}_{F M}$ is asymptotically stable and (8) is satisfied if there exist matrices $\mathcal{U}>0, \mathcal{V}>0, \mathcal{Q}_{1 j}>0$, 
$\mathcal{Q}_{3 j}>0, \mathcal{Q}_{11 j}>0, \mathcal{Q}_{13 j}>0, \mathcal{Q}_{21 j}>0, \mathcal{Q}_{23 j}>0, \mathcal{Q}_{2 j}, \mathcal{Q}_{12 j}, \mathcal{Q}_{22 j}, \mathcal{A}_{1 f}, \mathcal{A}_{2 f}, \mathcal{B}_{1 f}, \mathcal{B}_{2 f}$ and $\mathcal{C}_{f}$ such that, for $j=1,2, \ldots, s$, the following LMIs (23)-(26) hold:

$$
\begin{aligned}
& {\left[\begin{array}{cccc}
-\mathcal{U} & -\mathcal{V} & 0 & 0 \\
* & -\mathcal{V} & 0 & 0 \\
* & * & -I & 0 \\
* & * & * & -I \\
* & * & * & * \\
* & * & * & * \\
* & * & * & * \\
* & * & * & * \\
* & * & * & * \\
* & * & * & * \\
* & * & * & * \\
* & * & * & * \\
* & * & * & * \\
* & * & * & *
\end{array}\right.} \\
& 0 \quad \mathcal{U} A_{1 j}+\mathcal{B}_{1 f} C_{j} \\
& \mathcal{V} A_{1 j}+\mathcal{B}_{1 f} C_{j} \\
& \mathcal{A}_{1 f} \quad \mathcal{U} B_{1 j}+\mathcal{B}_{1 f} D_{j} \quad \mathcal{U} A_{d 1 j} \\
& \mathcal{A}_{1 f} \quad \mathcal{V} B_{1 j}+\mathcal{B}_{1 f} D_{j} \quad \mathcal{V} A_{d 1 j} \\
& -\mathcal{C}_{f} \\
& 0 \\
& 0 \quad 0 \\
& \begin{array}{c}
E_{j} \\
0
\end{array} \\
& \text { * } \quad \mathcal{Q}_{1 j}+\mathcal{Q}_{11 j}-\mathcal{U} \\
& \mathcal{Q}_{2 j}+\mathcal{Q}_{12 j}-\mathcal{V} \\
& 0 \quad 0 \\
& \mathcal{Q}_{3 j}+\mathcal{Q}_{13 j}-\mathcal{V} \\
& \begin{array}{ll}
0 & 0
\end{array} \\
& 0 \quad 0 \\
& * \quad * \\
& \text { * } \\
& \text { * } \\
& * \\
& * \\
& * \\
& * \\
& * \\
& * \\
& \begin{array}{ccc}
0 & \mathcal{U} A_{2 j}+\mathcal{B}_{2 f} C_{j} & \mathcal{A}_{2 f} \\
0 & \mathcal{V} A_{2 j}+\mathcal{B}_{2 f} C_{j} & \mathcal{A}_{2 f} \\
0 & 0 & 0 \\
0 & E_{j} & -\mathcal{C}_{f} \\
0 & 0 & 0 \\
0 & 0 & 0 \\
0 & 0 & 0 \\
-\mathcal{Q}_{12 j} & 0 & 0 \\
-\mathcal{Q}_{13 j} & 0 & 0 \\
* & \mathcal{Q}_{21 j}-\mathcal{Q}_{1 j} & \mathcal{Q}_{22 j}-\mathcal{Q}_{2 j} \\
* & * & \mathcal{Q}_{23 j}-\mathcal{Q}_{3 j} \\
* & * & * \\
* & * & * \\
* & * & *
\end{array} \\
& \mathcal{U} B_{2 j}+\mathcal{B}_{2 f} D_{j} \quad \mathcal{U} A_{d 2 j} \quad 0 \\
& \mathcal{V} B_{2 j}+\mathcal{B}_{2 f} D_{j} \quad \mathcal{V} A_{d 2 j} \quad 0 \\
& * \quad-\mathcal{Q}_{11 j} \\
& \text { * * } \\
& \text { * } \quad * \\
& * \quad * \\
& * \quad * \\
& * \quad * \\
& \text { * } \quad * \\
& 0 \quad 0 \quad 0 \\
& \begin{array}{lll}
0 & 0 & 0
\end{array} \\
& 0 \quad 0 \quad 0 \\
& \begin{array}{lll}
0 & 0 & 0
\end{array} \\
& \begin{array}{lll}
0 & 0 & 0 \\
0 & 0 & 0
\end{array} \mid<0 \\
& \begin{array}{lll}
0 & 0 & 0
\end{array} \\
& \begin{array}{lll}
0 & 0 & 0
\end{array} \\
& \begin{array}{lll}
0 & 0 & 0
\end{array} \\
& \begin{array}{lrr}
-\gamma^{2} I & 0 & 0
\end{array} \\
& * \quad-\mathcal{Q}_{21 j}-\mathcal{Q}_{22 j} \\
& \text { * } \quad * \quad-\mathcal{Q}_{23 j} \\
& {\left[\begin{array}{cc}
\mathcal{Q}_{1 j} & \mathcal{Q}_{2 j} \\
* & \mathcal{Q}_{3 j}
\end{array}\right]>0} \\
& {\left[\begin{array}{cc}
\mathcal{Q}_{11 j} & \mathcal{Q}_{12 j} \\
* & \mathcal{Q}_{13 j}
\end{array}\right]>0} \\
& {\left[\begin{array}{cc}
\mathcal{Q}_{21 j} & \mathcal{Q}_{22 j} \\
* & \mathcal{Q}_{23 j}
\end{array}\right]>0}
\end{aligned}
$$

Moreover, a desired $\mathcal{H}_{\infty}$ filter is given in the form of (4) with parameters can be computed from (14).

\section{B. Norm-Bounded Uncertain Case}

An alternative way of dealing with uncertain systems is to assume that the deviation of the system parameters from their nominal values is norm-bounded, which has also been widely used in the robust control and filtering problems.

Assumption 4: The matrices $A_{1}, A_{2}, A_{d 1}, A_{d 2}, B_{1}, B_{2}, C, D$ and $E$ of system $\Sigma_{F M}$ in (1) are assumed to 
have the following form

$$
\begin{aligned}
& A_{1}=\hat{A}_{1}+\Delta A_{1}, \quad B_{1}=\hat{B}_{1}+\Delta B_{1}, \quad A_{d 1}=\hat{A}_{d 1}+\Delta A_{d 1}, \\
& A_{2}=\hat{A}_{2}+\Delta A_{2}, \quad B_{2}=\hat{B}_{2}+\Delta B_{2}, \quad A_{d 2}=\hat{A}_{d 2}+\Delta A_{d 2}, \\
& C=\hat{C}+\Delta C, \quad D=\hat{D}+\Delta D, \quad E=\hat{E}+\Delta E
\end{aligned}
$$

where $\hat{A}_{1}, \hat{A}_{2}, \hat{A}_{d 1}, \hat{A}_{d 2}, \hat{B}_{1}, \hat{B}_{2}, \hat{C}, \hat{D}$ and $\hat{E}$ are known constant matrices with appropriate dimensions. $\Delta A_{1}$, $\Delta A_{2}, \Delta A_{d 1}, \Delta A_{d 2}, \Delta B_{1}, \Delta B_{2}, \Delta C, \Delta D$ and $\Delta E$ are real-valued time-varying matrix functions representing norm-bounded parameter uncertainties satisfying

$$
\left[\begin{array}{ccc}
\Delta A_{1} & \Delta B_{1} & \Delta A_{d 1} \\
\Delta A_{2} & \Delta B_{2} & \Delta A_{d 2} \\
\Delta C & \Delta D & \Delta E
\end{array}\right]=\left[\begin{array}{l}
M_{1} \\
M_{2} \\
M_{3}
\end{array}\right] \Delta\left[\begin{array}{lll}
N_{1} & N_{2} & N_{3}
\end{array}\right]
$$

where $\Delta_{i, j}$ is a real uncertain matrix function with Lebesgue measurable elements satisfying $\Delta^{T} \Delta \leq I$, and $M_{1}, M_{2}, M_{3}, N_{1}, N_{2}$ and $N_{3}$ are known real constant matrices of appropriate dimensions.

Before proceeding further, we give the following lemma which will be used in the proof of this subsection (see, for instance, $[26]$ ).

Lemma 2: Given appropriately dimensioned matrices $\Sigma_{1}, \Sigma_{2}$ and $\Sigma_{3}$ with $\Sigma_{1}^{T}=\Sigma_{1}$. Then

$$
\Sigma_{1}+\Sigma_{2} \Omega \Sigma_{3}+\Sigma_{3}^{T} \Omega^{T} \Sigma_{2}^{T}<0
$$

holds for all $\Omega$ satisfying $\Omega^{T} \Omega \leq I$ if and only if for some $\epsilon>0$

$$
\Sigma_{1}+\epsilon^{-1} \Sigma_{2} \Sigma_{2}^{T}+\epsilon \Sigma_{3}^{T} \Sigma_{3}<0 .
$$

We now present the robust $\mathcal{H}_{\infty}$ filtering result for the system $\Sigma_{F M}$ in (1) with norm-bounded uncertainties in the following theorem.

Theorem 4: Consider the 2-D state-delayed system $\Sigma_{F M}$ in (1) with Assumption 4 and let $\gamma>0$ be a prescribed constant scalar. Then there exists a full-order filter $\hat{\Sigma}_{F M}$ in the form of (4) such that the filtering error system $\tilde{\Sigma}_{F M}$ is asymptotically stable and (8) is satisfied if there exist matrices $\mathcal{U}>0, \mathcal{V}>0, \mathcal{Q}_{1}>0$, $\mathcal{Q}_{3}>0, \mathcal{Q}_{11}>0, \mathcal{Q}_{13}>0, \mathcal{Q}_{21}>0, \mathcal{Q}_{23}>0, \mathcal{Q}_{2}, \mathcal{Q}_{12}, \mathcal{Q}_{22}, \mathcal{A}_{1 f}, \mathcal{A}_{2 f}, \mathcal{B}_{1 f}, \mathcal{B}_{2 f}$ and $\mathcal{C}_{f}$, scalars $\epsilon_{j}>0$ $(j=1,2, \ldots, 6)$ such that the LMIs (11)-(13) and LMI (29) (shown at the top of the next page) hold. In (29), some notations are defined as follows:

$$
\begin{aligned}
\Psi_{55} & \triangleq \mathcal{Q}_{1}+\mathcal{Q}_{11}-\mathcal{U}+\left(\epsilon_{1}+\epsilon_{3}\right) N_{1}^{T} N_{1}+\epsilon_{5} N_{3}^{T} N_{3}, \quad \Psi_{56} \triangleq \mathcal{Q}_{2}+\mathcal{Q}_{12}-\mathcal{V} \\
\Psi_{57} & \triangleq\left(\epsilon_{1}+\epsilon_{3}\right) N_{1}^{T} N_{2}, \quad \Psi_{66} \triangleq \mathcal{Q}_{3}+\mathcal{Q}_{13}-\mathcal{V}, \quad \Psi_{77} \triangleq\left(\epsilon_{1}+\epsilon_{3}\right) N_{2}^{T} N_{2}-\gamma^{2} I \\
\Psi_{88} & \triangleq \epsilon_{1} N_{3}^{T} N_{3}-\mathcal{Q}_{11}, \quad \Psi_{1011} \triangleq \mathcal{Q}_{22}-\mathcal{Q}_{2}, \quad \Psi_{1111} \triangleq \mathcal{Q}_{23}-\mathcal{Q}_{3} \\
\Psi_{1010} & \triangleq \mathcal{Q}_{21}-\mathcal{Q}_{1}+\left(\epsilon_{2}+\epsilon_{4}\right) N_{1}^{T} N_{1}+\epsilon_{6} N_{3}^{T} N_{3}, \quad \Psi_{1012} \triangleq\left(\epsilon_{2}+\epsilon_{4}\right) N_{1}^{T} N_{2} \\
\Psi_{1212} & \triangleq\left(\epsilon_{2}+\epsilon_{4}\right) N_{2}^{T} N_{2}-\gamma^{2} I, \quad \Psi_{1313} \triangleq \epsilon_{2} N_{3}^{T} N_{3}-\mathcal{Q}_{21}
\end{aligned}
$$


Moreover, a desired $\mathcal{H}_{\infty}$ filter is given in the form of (4) with parameters can be computed from (14).

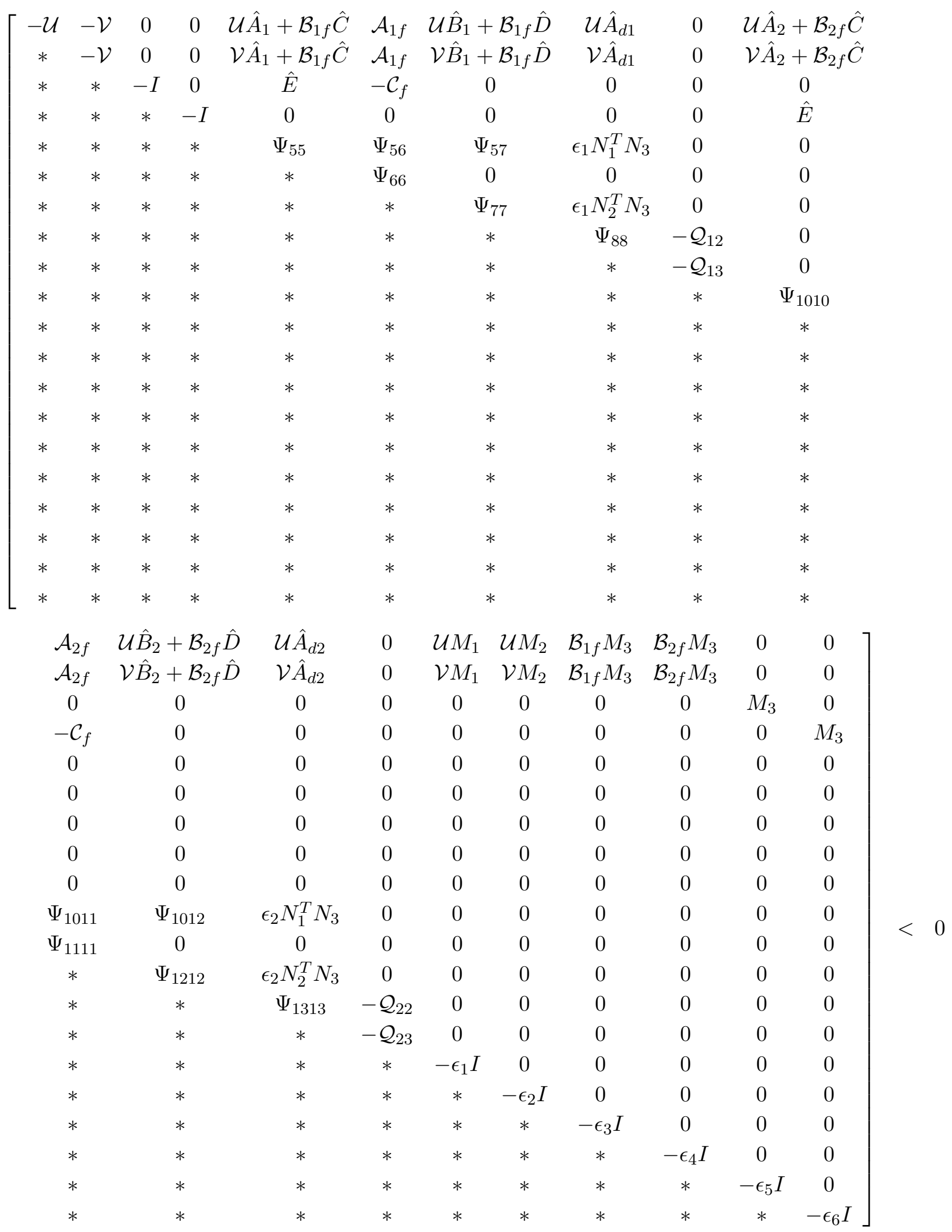


Proof: With the result of Theorem 2, we substitute the norm-bounded uncertain matrices $A_{1}, A_{2}, A_{d 1}$, $A_{d 2}, B_{1}, B_{2}, C, D$ and $E$ defined in (27) into (10) and obtain (28) where

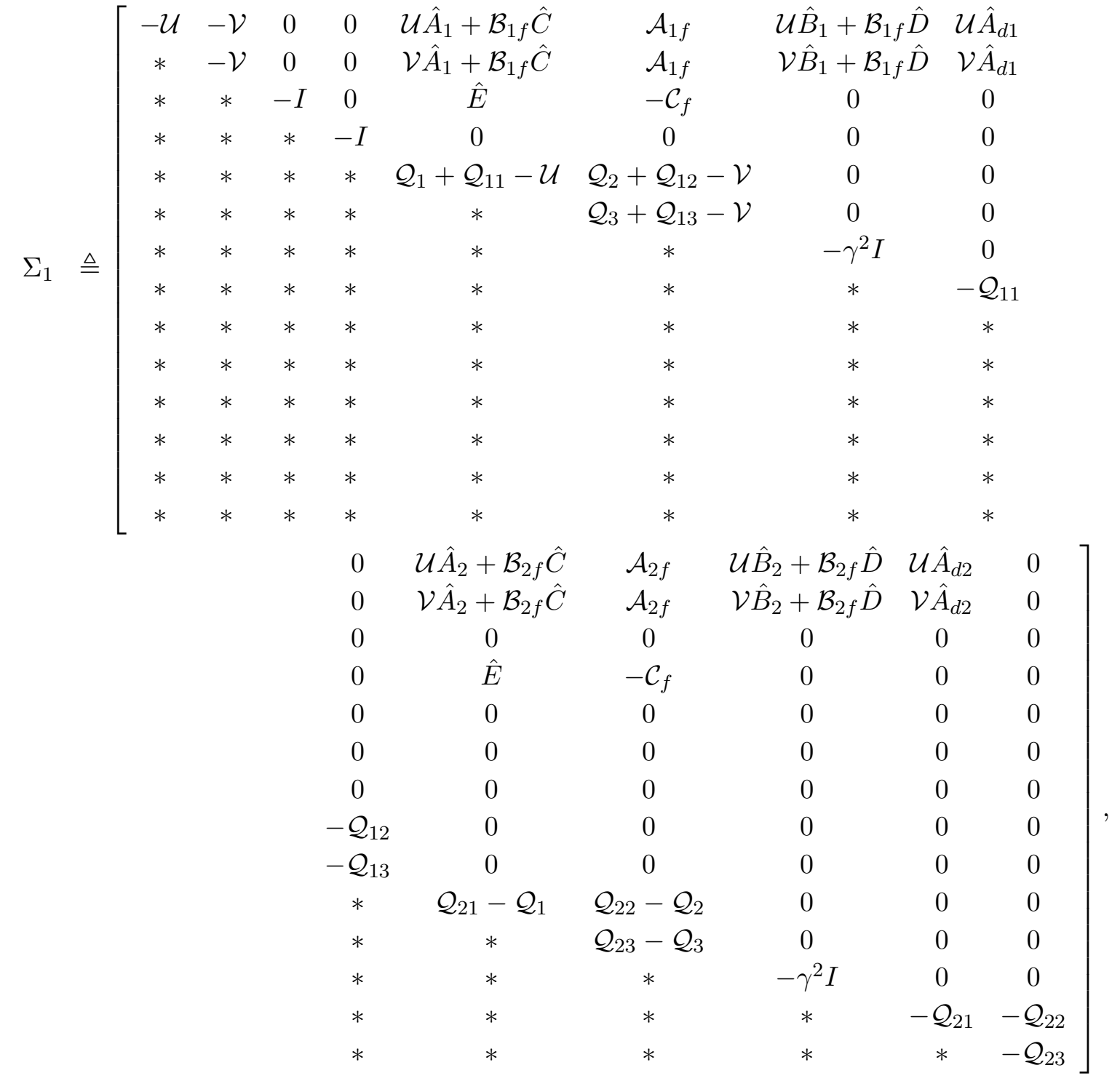

$$
\begin{aligned}
& \Sigma_{2} \triangleq\left[\begin{array}{cccccccccccccc}
M_{1}^{T} \mathcal{U} & M_{1}^{T} \mathcal{V} & 0 & 0 & 0 & 0 & 0 & 0 & 0 & 0 & 0 & 0 & 0 & 0 \\
M_{2}^{T} \mathcal{U} & M_{2}^{T} \mathcal{V} & 0 & 0 & 0 & 0 & 0 & 0 & 0 & 0 & 0 & 0 & 0 & 0 \\
M_{3}^{T} \mathcal{B}_{1 f}^{T} & M_{3}^{T} \mathcal{B}_{1 f}^{T} & 0 & 0 & 0 & 0 & 0 & 0 & 0 & 0 & 0 & 0 & 0 & 0 \\
M_{3}^{T} \mathcal{B}_{2 f}^{T} & M_{3}^{T} \mathcal{B}_{2 f}^{T} & 0 & 0 & 0 & 0 & 0 & 0 & 0 & 0 & 0 & 0 & 0 & 0 \\
0 & 0 & M_{3}^{T} & 0 & 0 & 0 & 0 & 0 & 0 & 0 & 0 & 0 & 0 & 0 \\
0 & 0 & 0 & M_{3}^{T} & 0 & 0 & 0 & 0 & 0 & 0 & 0 & 0 & 0 & 0
\end{array}\right]^{T} \\
& \Sigma_{3} \triangleq\left[\begin{array}{cccccccccccccc}
0 & 0 & 0 & 0 & N_{1} & 0 & N_{2} & N_{3} & 0 & 0 & 0 & 0 & 0 & 0 \\
0 & 0 & 0 & 0 & 0 & 0 & 0 & 0 & 0 & N_{1} & 0 & N_{2} & N_{3} & 0 \\
0 & 0 & 0 & 0 & N_{1} & 0 & N_{2} & 0 & 0 & 0 & 0 & 0 & 0 & 0 \\
0 & 0 & 0 & 0 & 0 & 0 & 0 & 0 & 0 & N_{1} & 0 & N_{2} & 0 & 0 \\
0 & 0 & 0 & 0 & N_{3} & 0 & 0 & 0 & 0 & 0 & 0 & 0 & 0 & 0 \\
0 & 0 & 0 & 0 & 0 & 0 & 0 & 0 & 0 & N_{3} & 0 & 0 & 0 & 0
\end{array}\right], \Omega \triangleq\left[\begin{array}{cccccc}
\Delta & 0 & 0 & 0 & 0 & 0 \\
0 & \Delta & 0 & 0 & 0 & 0 \\
0 & 0 & \Delta & 0 & 0 & 0 \\
0 & 0 & 0 & \Delta & 0 & 0 \\
0 & 0 & 0 & 0 & \Delta & 0 \\
0 & 0 & 0 & 0 & 0 & \Delta
\end{array}\right] .
\end{aligned}
$$

By invoking Lemma 1 together with a Schur complement operation, (28) holds if and only if (29) holds, which completes the proof. 


\section{Illustrative Example}

In a real world, some dynamical processes in gas absorption, water stream heating and air drying can be described by the Darboux equation with time-delays [16]:

$$
\begin{aligned}
\frac{\partial^{2} s(x, t)}{\partial x \partial t} & =a_{11} \frac{\partial s(x, t)}{\partial t}+a_{12} \frac{\partial s\left(x, t-\tau_{1}\right)}{\partial t}+a_{21} \frac{\partial s(x, t)}{\partial x}+a_{22} \frac{\partial s\left(x, t-\tau_{1}\right)}{\partial x}+a_{0} s(x, t)+b f(x, t) \\
y(x, t) & =c_{1} s(x, t)+c_{2}\left[\frac{\partial s(x, t)}{\partial t}-a_{21} s(x, t)\right]
\end{aligned}
$$

where $s(x, t)$ is an unknown function at $x$ (space) $\in\left[0, x_{f}\right]$ and $t$ (time) $\in[0, \infty), \tau_{1}$ is the time delay, $a_{0}, a_{11}$, $a_{12}, a_{21}, a_{22}, b, c_{1}$ and $c_{2}$ are real coefficients, $f(x, t)$ is the input function, and $y(x, t)$ is the measured output.

Note that (30)-(31) is a partial differential equation (PDE) and, in practice, it is often desired to predict the unknown state function $s(x, t)$ through the available measurement $y(x, t)$, which renders the filtering problem. Similar to the technique used in [5], we define

$$
\begin{gathered}
r(x, t):=\frac{\partial s(x, t)}{\partial t}-a_{21} s(x, t)+\frac{\partial s\left(x, t-\tau_{1}\right)}{\partial t}-a_{22} s\left(x, t-\tau_{1}\right) \\
x_{1}(i, j):=r(i, j):=r(i \Delta x, j \Delta t), \quad x_{2}(i, j):=s(i, j):=s(i \Delta x, j \Delta t),
\end{gathered}
$$

and then the PDE model (30)-(31) can be converted into the form of a state delayed 2-D system $\Sigma_{F M}$ in (1).

As discussed in [5], the discrepancy between the PDE model and its 2-D difference approximation depends on the step sizes $\Delta x$ and $\Delta t$ which may be treated as uncertainty in the difference model. Obviously, the smaller the step sizes $\Delta x$ and $\Delta t$, the closer between the PDE model and the difference model.

Now, subject to the selection of the parameters $a_{0}, a_{11}, a_{12}, a_{21}, a_{22}, b, c_{1}$ and $c_{2}$, we let the system matrices be given as follows:

$$
\begin{aligned}
A_{1}=\left[\begin{array}{cc}
0.3 & 0 \\
0.2 & 0.1+0.02 \delta
\end{array}\right], \quad B_{1}=\left[\begin{array}{c}
0.3 \\
0.5+0.01 \delta
\end{array}\right], \quad A_{d 1}=\left[\begin{array}{cc}
0.2 & 0 \\
0 & 0.1+0.02 \delta
\end{array}\right] \\
A_{2}=\left[\begin{array}{cc}
0.1 & 0 \\
0.2 & 0.2+0.02 \delta
\end{array}\right], \quad B_{2}=\left[\begin{array}{c}
0.2 \\
0.4+0.01 \delta
\end{array}\right], \quad A_{d 2}=\left[\begin{array}{cc}
0 & 0.1 \\
0 & 0.2+0.02 \delta
\end{array}\right] \\
C=\left[\begin{array}{cc}
1.0 & 0 \\
1.0 & 0.6+0.02 \delta
\end{array}\right], \quad D=\left[\begin{array}{c}
0 \\
0.3+0.01 \delta
\end{array}\right], \quad E=\left[\begin{array}{cc}
-1.0 & 1.0 \\
0 & -0.8+0.02 \delta
\end{array}\right]
\end{aligned}
$$

First, we assume that the system matrices are perfectly known, that is, $\delta=0$. Solving the LMIs condition obtained in Theorem 2 by applying the well-developed LMI-Toolbox in the MATLAB environment directly, we obtain that the minimum $\gamma$ is $\gamma^{*}=3.8207$ and

$$
\begin{aligned}
& A_{1 f}=\left[\begin{array}{cc}
-0.0117 & 0.0086 \\
0.0086 & -0.0063
\end{array}\right], B_{1 f}=\left[\begin{array}{cc}
-2.1209 & 1.0000 \\
1.5539 & -0.7343
\end{array}\right] \\
& A_{2 f}=\left[\begin{array}{cc}
-0.0101 & 0.0074 \\
0.0072 & -0.0053
\end{array}\right], B_{2 f}=\left[\begin{array}{cc}
-1.5607 & 1.9339 \\
1.1429 & -1.4179
\end{array}\right], C_{f}=\left[\begin{array}{cc}
1.3918 & -1.0222 \\
-1.1895 & 0.8761
\end{array}\right] .
\end{aligned}
$$

Now, we assume $|\delta| \leq 1$, that is, the system considered has parameter uncertainties. As mentioned in the previous section, there are two types of parameter uncertainties, namely, polytopic uncertainties and normbounded uncertainties. In the following, firstly, we consider the polytopic uncertainties case. In this case, according to Assumption 3, the parameter uncertainties can be represented by a two-vertex polytope. Using Theorem 3, the minimum $\gamma$ obtained is $\gamma^{*}=5.4379$, and the obtained filter parameter matrices are given as 
follows:

$$
\begin{aligned}
& A_{1 f}=\left[\begin{array}{cc}
-0.0661 & 0.0378 \\
0.0375 & -0.0215
\end{array}\right], B_{1 f}=\left[\begin{array}{cc}
-6.3037 & 2.3277 \\
3.6136 & -1.3374
\end{array}\right] \\
& A_{2 f}=\left[\begin{array}{cc}
-0.0428 & 0.0245 \\
0.0237 & -0.0136
\end{array}\right], B_{2 f}=\left[\begin{array}{cc}
-4.6924 & 4.1119 \\
2.6911 & -2.3608
\end{array}\right], C_{f}=\left[\begin{array}{cc}
1.8108 & -1.0445 \\
-1.5350 & 0.8889
\end{array}\right] .
\end{aligned}
$$

Finally, we consider the norm-bounded uncertainties case, and the uncertainties are characterized as follows according to Assumption 4:

$$
\begin{aligned}
& \hat{A}_{1}=\left[\begin{array}{cc}
0.3 & 0 \\
0.2 & 0.1
\end{array}\right], \quad \hat{A}_{2}=\left[\begin{array}{cc}
0.1 & 0 \\
0.2 & 0.2
\end{array}\right], \quad \hat{A}_{d 1}=\left[\begin{array}{cc}
0.2 & 0 \\
0 & 0.1
\end{array}\right], \quad \hat{A}_{d 2}=\left[\begin{array}{cc}
0 & 0.1 \\
0 & 0.2
\end{array}\right] \\
& \hat{B}_{1}=\left[\begin{array}{l}
0.3 \\
0.5
\end{array}\right], \quad \hat{B}_{2}=\left[\begin{array}{l}
0.2 \\
0.4
\end{array}\right], \quad \hat{C}=\left[\begin{array}{cc}
1.0 & 0 \\
1.0 & 0.6
\end{array}\right], \quad \hat{D}=\left[\begin{array}{c}
0 \\
0.3
\end{array}\right], \quad \hat{E}=\left[\begin{array}{cc}
-1.0 & 1.0 \\
0 & -0.8
\end{array}\right] \\
& M_{1}=M_{2}=M_{3}=\left[\begin{array}{l}
0 \\
1
\end{array}\right], \quad N_{1}=N_{3}=\left[\begin{array}{ll}
0 & 0.02
\end{array}\right], \quad N_{2}=0.02 .
\end{aligned}
$$

Using Theorem 4, the minimum $\gamma$ is obtained as $\gamma^{*}=5.2074$, and the obtained filter parameter matrices are given as follows:

$$
\begin{aligned}
& A_{1 f}=\left[\begin{array}{cc}
0.6830 & -0.4439 \\
-0.4496 & 0.2917
\end{array}\right], B_{1 f}=\left[\begin{array}{cc}
-2.2071 & 0.4063 \\
1.4350 & -0.2651
\end{array}\right] \\
& A_{2 f}=\left[\begin{array}{cc}
1.8433 & -1.2020 \\
-1.2065 & 0.7864
\end{array}\right], B_{2 f}=\left[\begin{array}{cc}
4.4037 & 0.6876 \\
-2.8895 & -0.4487
\end{array}\right], C_{f}=\left[\begin{array}{cc}
1.6334 & -1.0734 \\
-1.4913 & 0.9842
\end{array}\right]
\end{aligned}
$$

Let the disturbance input $\omega(i, j)$ be

$$
\omega(i, j)= \begin{cases}0.05, & 3 \leq i, j \leq 19 \\ 0, & \text { otherwise }\end{cases}
$$

In the following, we shall show the usefulness of the designed $\mathcal{H}_{\infty}$ filters by presenting simulation results. To show the asymptotic stability of the filtering error system, let the initial and boundary conditions be

$$
x(0, i)=x(i, 0)= \begin{cases}{\left[\begin{array}{ll}
1 & 1.5
\end{array}\right]^{T},} & 0 \leq i \leq 15 \\
{\left[\begin{array}{ll}
0 & 0
\end{array}\right]^{T},} & i>15\end{cases}
$$

The state response of the designed $\mathcal{H}_{\infty}$ filter in (4) with (32) are given in Figures 1 and 2, and Figures 3 and 4 are the error response for $e(i, j)$. It can be seen from Figures 3 and 4 that the designed $\mathcal{H}_{\infty}$ filter guarantees that $e(i, j)$ converges to zero under the above conditions.

\section{Concluding Remarks}

In this paper, the problem of robust $\mathcal{H}_{\infty}$ filtering for a class of 2-D delayed systems has been studied. Some sufficient conditions have been proposed for the existences of robust $\mathcal{H}_{\infty}$ filter in terms of LMI. The designed robust $\mathcal{H}_{\infty}$ filter guarantees robust asymptotic stability and a prescribed $\mathcal{H}_{\infty}$ performance of the filtering error system, and the desired filter can be found by solving a convex optimization problem. In addition, the obtained results have been further extended to more general cases where the system matrices also contain 
uncertain parameters. The most frequently used methods of dealing with parameter uncertainties, including polytopic and norm-bounded characterizations, have been taken into consideration. An illustrative example has been presented to demonstrate the effectiveness of the proposed methods. One of the future research topics would be the further investigation on the time-varying time delays case [17].

\section{APPENDIX}

The proof of Theorem 1.

Proof: First, let us establish the asymptotic stability of the error system $\tilde{\Sigma}_{F M}$ in $(5)$ with $\omega_{i, j} \equiv 0$. Denote

$$
\begin{aligned}
V_{11}(i, j) \triangleq & \xi_{11}^{T}(i, j) P \xi_{11}(i, j)+\sum_{\kappa=-d_{1}}^{-1} \xi_{\kappa 1}^{T}(i+1, j) Q_{1} \xi_{\kappa 1}(i+1, j) \\
& +\sum_{\kappa=-d_{2}}^{-1} \xi_{1 \kappa}^{T}(i, j+1) Q_{2} \xi_{1 \kappa}(i, j+1) \\
V_{d 1}(i, j) \triangleq & \xi_{01}^{T}(i, j)(P-Q) \xi_{01}(i, j)+\sum_{\kappa=-d_{1}}^{-1} \xi_{\kappa 1}^{T}(i, j) Q_{1} \xi_{\kappa 1}(i, j) \\
V_{d 2}(i, j) \triangleq & \xi_{10}^{T}(i, j) Q \xi_{10}(i, j)+\sum_{\kappa=-d_{2}}^{-1} \xi_{1 \kappa}^{T}(i, j) Q_{2} \xi_{1 \kappa}(i, j)
\end{aligned}
$$

Consider the increment $\Delta V(i, j)$ given by

$$
\Delta V(i, j) \triangleq V_{11}(i, j)-V_{01}(i, j)-V_{10}(i, j)
$$

Then, along the solution of the filtering error system $\tilde{\Sigma}_{F M}$, we have

$$
\begin{aligned}
\Delta V(i, j)= & {\left[\tilde{A}_{1} \xi(i, j+1)+\tilde{A}_{2} \xi(i+1, j)+\tilde{A}_{d 1} \xi\left(i-d_{1}, j+1\right)+\tilde{A}_{d 2} \xi\left(i+1, j-d_{2}\right)\right]^{T} P } \\
& \times\left[\tilde{A}_{1} \xi(i, j+1)+\tilde{A}_{2} \xi(i+1, j)+\tilde{A}_{d 1} \xi\left(i-d_{1}, j+1\right)+\tilde{A}_{d 2} \xi\left(i+1, j-d_{2}\right)\right] \\
& -\xi^{T}(i, j+1)\left(P-Q-Q_{1}\right) \xi(i, j+1)-\xi^{T}(i+1, j)\left(Q-Q_{2}\right) \xi(i+1, j) \\
& -\xi^{T}\left(i-d_{1}, j+1\right) Q_{1} \xi\left(i-d_{1}, j+1\right)-\xi^{T}\left(i+1, j-d_{2}\right) Q_{2} \xi\left(i+1, j-d_{2}\right) \\
\triangleq & \eta^{T}(i, j) \Psi \eta(i, j),
\end{aligned}
$$

where $\eta(i, j) \triangleq\left[\xi^{T}(i, j+1), \xi^{T}(i+1, j), \xi^{T}\left(i-d_{1}, j+1\right), \xi^{T}\left(i+1, j-d_{2}\right)\right]^{T}$ and

$$
\Psi \triangleq\left[\begin{array}{c}
\tilde{A}_{1}^{T} \\
\tilde{A}_{2}^{T} \\
\tilde{A}_{d 1}^{T} \\
\tilde{A}_{d 2}^{T}
\end{array}\right] P\left[\begin{array}{llll}
\tilde{A}_{1} & \tilde{A}_{2} & \tilde{A}_{d 1} & \tilde{A}_{d 2}
\end{array}\right]-\left[\begin{array}{cccc}
P-Q-Q_{1} & 0 & 0 & 0 \\
* & Q-Q_{2} & 0 & 0 \\
* & * & Q_{1} & 0 \\
* & * & * & Q_{2}
\end{array}\right] .
$$

By Schur complement [2], LMI (9) implies $\Psi<0$. It follows from Lemma 1 (Theorem 3 of [19]) that the 2-D filtering error system $\tilde{\Sigma}_{F M}$ in $(5)$ with $\omega(i, j) \equiv 0$ is asymptotically stable.

Now, to establish the $\mathcal{H}_{\infty}$ performance for the filtering error system $\tilde{\Sigma}_{F M}$ in (5), introduce the following index:

$$
\mathcal{J} \triangleq \Delta V(i, j)+\bar{e}^{T}(i, j) \bar{e}(i, j)-\gamma^{2} \bar{\omega}^{T}(i, j) \bar{\omega}(i, j)
$$


where

$$
\begin{aligned}
\Delta V(i, j)= & {\left[\tilde{A}_{1} \xi(i, j+1)+\tilde{A}_{2} \xi(i+1, j)+\tilde{A}_{d 1} \xi\left(i-d_{1}, j+1\right)+\tilde{A}_{d 2} \xi\left(i+1, j-d_{2}\right)\right.} \\
& \left.+\tilde{B}_{1} \omega(i, j+1)+\tilde{B}_{2} \omega(i+1, j)\right]^{T} P\left[\tilde{A}_{1} \xi(i, j+1)+\tilde{A}_{2} \xi(i+1, j)\right. \\
& \left.+\tilde{A}_{d 1} \xi\left(i-d_{1}, j+1\right)+\tilde{A}_{d 2} \xi\left(i+1, j-d_{2}\right)+\tilde{B}_{1} \omega(i, j+1)+\tilde{B}_{2} \omega(i+1, j)\right] \\
& -\xi^{T}(i, j+1)\left(P-Q-Q_{1}\right) \xi(i, j+1)-\xi^{T}(i+1, j)\left(Q-Q_{2}\right) \xi(i+1, j) \\
& -\xi^{T}\left(i-d_{1}, j+1\right) Q_{1} \xi\left(i-d_{1}, j+1\right)-\xi^{T}\left(i+1, j-d_{2}\right) Q_{2} \xi\left(i+1, j-d_{2}\right)
\end{aligned}
$$

According to the stability of the system, we have

$$
\begin{aligned}
& \mathcal{J}=\left[\tilde{A}_{1} \xi(i, j+1)+\tilde{A}_{2} \xi(i+1, j)+\tilde{A}_{d 1} \xi\left(i-d_{1}, j+1\right)+\tilde{A}_{d 2} \xi\left(i+1, j-d_{2}\right)\right. \\
& \left.+\tilde{B}_{1} \omega(i, j+1)+\tilde{B}_{2} \omega(i+1, j)\right]^{T} P\left[\tilde{A}_{1} \xi(i, j+1)+\tilde{A}_{2} \xi(i+1, j)\right. \\
& \left.+\tilde{A}_{d 1} \xi\left(i-d_{1}, j+1\right)+\tilde{A}_{d 2} \xi\left(i+1, j-d_{2}\right)+\tilde{B}_{1} \omega(i, j+1)+\tilde{B}_{2} \omega(i+1, j)\right] \\
& -\xi^{T}(i, j+1)\left(P-Q-Q_{1}\right) \xi(i, j+1)-\xi^{T}(i+1, j)\left(Q-Q_{2}\right) \xi(i+1, j) \\
& -\xi^{T}\left(i-d_{1}, j+1\right) Q_{1} \xi\left(i-d_{1}, j+1\right)-\xi^{T}\left(i+1, j-d_{2}\right) Q_{2} \xi\left(i+1, j-d_{2}\right) \\
& +\xi^{T}(i, j+1) \tilde{C}^{T} \tilde{C} \xi(i, j+1)+\xi^{T}(i+1, j) \tilde{C}^{T} \tilde{C} \xi(i+1, j) \\
& -\gamma^{2} \omega^{T}(i, j+1) \omega(i, j+1)-\gamma^{2} \omega^{T}(i+1, j) \omega(i+1, j) \\
& =\left[\tilde{A}_{1} \xi(i, j+1)+\tilde{A}_{2} \xi(i+1, j)+\tilde{A}_{d 1} \xi\left(i-d_{1}, j+1\right)+\tilde{A}_{d 2} \xi\left(i+1, j-d_{2}\right)\right]^{T} P \\
& \times\left[\tilde{A}_{1} \xi(i, j+1)+\tilde{A}_{2} \xi(i+1, j)+\tilde{A}_{d 1} \xi\left(i-d_{1}, j+1\right)+\tilde{A}_{d 2} \xi\left(i+1, j-d_{2}\right)\right] \\
& -\xi^{T}(i, j+1)\left(P-Q-Q_{1}\right) \xi(i, j+1)-\xi^{T}(i+1, j)\left(Q-Q_{2}\right) \xi(i+1, j) \\
& -\xi^{T}\left(i-d_{1}, j+1\right) Q_{1} \xi\left(i-d_{1}, j+1\right)-\xi^{T}\left(i+1, j-d_{2}\right) Q_{2} \xi\left(i+1, j-d_{2}\right) \\
& +\xi^{T}(i, j+1) \tilde{C}^{T} \tilde{C} \xi(i, j+1)+\xi^{T}(i+1, j) \tilde{C}^{T} \tilde{C} \xi(i+1, j) \\
& +2\left[\tilde{A}_{1} \xi(i, j+1)+\tilde{A}_{2} \xi(i+1, j)+\tilde{A}_{d 1} \xi\left(i-d_{1}, j+1\right)+\tilde{A}_{d 2} \xi\left(i+1, j-d_{2}\right)\right]^{T} P \\
& \times\left[\tilde{B}_{1} \omega(i, j+1)+\tilde{B}_{2} \omega(i+1, j)\right] \\
& -\left\{\gamma^{2} \omega^{T}(i, j+1) \omega(i, j+1)+\gamma^{2} \omega^{T}(i+1, j) \omega(i+1, j)\right. \\
& \left.-\left[\tilde{B}_{1} \omega(i, j+1)+\tilde{B}_{2} \omega(i+1, j)\right]^{T} P\left[\tilde{B}_{1} \omega(i, j+1)+\tilde{B}_{2} \omega(i+1, j)\right]\right\} \\
& \triangleq \eta^{T}(i, j) \Pi \eta(i, j)+2 \eta^{T}(i, j) \Omega \bar{\omega}(i, j)-\bar{\omega}^{T}(i, j) \Phi \bar{\omega}(i, j) \\
& =\eta^{T}(i, j) \Pi \eta(i, j)+\eta^{T}(i, j) \Omega \Phi^{-1} \Omega^{T} \eta(i, j)-\eta^{T}(i, j) \Omega \Phi^{-1} \Omega^{T} \eta(i, j) \\
& +2 \eta^{T}(i, j) \Omega \bar{\omega}(i, j)-\bar{\omega}^{T}(i, j) \Phi \bar{\omega}(i, j) \\
& =\eta^{T}(i, j)\left(\Pi+\Omega \Phi^{-1} \Omega^{T}\right) \eta(i, j)-\left[\eta^{T}(i, j) \Omega \Phi^{-1} \Omega^{T} \eta(i, j)\right. \\
& \left.-2 \eta^{T}(i, j) \Omega \bar{\omega}(i, j)+\bar{\omega}^{T}(i, j) \Phi \bar{\omega}(i, j)\right] \\
& =\eta^{T}(i, j) \Sigma \eta(i, j)-\left[\bar{\omega}(i, j)-\Phi^{-1} \Omega^{T} \eta(i, j)\right]^{T} \Phi\left[\bar{\omega}(i, j)-\Phi^{-1} \Omega^{T} \eta(i, j)\right] \\
& \triangleq \eta^{T}(i, j) \Sigma \eta(i, j)-\mu^{T}(i, j) \mu(i, j)
\end{aligned}
$$


where $\eta(i, j)$ is defined in $(35), \mu(i, j) \triangleq \Phi^{\frac{1}{2}}\left[\bar{\omega}(i, j)-\Phi^{-1} \Omega^{T} \eta(i, j)\right]$ and

$$
\begin{aligned}
\Pi \triangleq & {\left[\begin{array}{c}
\tilde{A}_{1}^{T} \\
\tilde{A}_{2}^{T} \\
\tilde{A}_{d 1}^{T} \\
\tilde{A}_{d 2}^{T}
\end{array}\right] P\left[\begin{array}{c}
\tilde{A}_{1}^{T} \\
\tilde{A}_{2}^{T} \\
\tilde{A}_{d 1}^{T} \\
\tilde{A}_{d 2}^{T}
\end{array}\right]^{T}+\left[\begin{array}{cccc}
Q+Q_{1}-P+\tilde{C}^{T} \tilde{C} & 0 & 0 & 0 \\
* & Q_{2}-Q+\tilde{C}^{T} \tilde{C} & 0 & 0 \\
* & * & -Q_{1} & 0 \\
* & * & * & -Q_{2}
\end{array}\right] } \\
\Omega \triangleq & {\left[\begin{array}{c}
\tilde{A}_{1}^{T} \\
\tilde{A}_{2}^{T} \\
\tilde{A}_{d 1}^{T} \\
\tilde{A}_{d 2}^{T}
\end{array}\right] P\left[\begin{array}{cc}
\tilde{B}_{1} & \tilde{B}_{2}
\end{array}\right], \quad \Phi \triangleq \gamma^{2} I-\left[\begin{array}{c}
\tilde{B}_{1}^{T} \\
\tilde{B}_{2}^{T}
\end{array}\right] P\left[\begin{array}{ll}
\tilde{B}_{1} & \tilde{B}_{2}
\end{array}\right], \quad \Sigma \triangleq \Pi+\Omega \Phi^{-1} \Omega^{T} }
\end{aligned}
$$

By Schur complement, LMI (9) implies $\Sigma<0$. This together with (36) and (38) yields

$$
\Delta V(i, j)+\bar{e}^{T}(i, j) \bar{e}(i, j)-\gamma^{2} \bar{\omega}^{T}(i, j) \bar{\omega}(i, j)<-\mu^{T}(i, j) \mu(i, j)
$$

Therefore we can sum both sides of (39) to obtain

$$
\sum_{i=0}^{\infty} \sum_{j=0}^{\infty}\left[\Delta V(i, j)+\bar{e}^{T}(i, j) \bar{e}(i, j)-\gamma^{2} \bar{\omega}^{T}(i, j) \bar{\omega}(i, j)\right]<-\sum_{i=0}^{\infty} \sum_{j=0}^{\infty} \mu^{T}(i, j) \mu(i, j)=-\|\mu(i, j)\|_{2}^{2}
$$

For any integers $p, q>0$, it follows from (34) that

$$
\begin{aligned}
\sum_{i=0}^{p} \sum_{j=0}^{q} \Delta V(i, j)= & \sum_{j=0}^{q}\left[\xi_{11}^{T}(p, j)(P-Q) \xi_{11}(p, j)-\xi_{01}^{T}(0, j)(P-Q) \xi_{01}(0, j)\right] \\
& +\sum_{i=0}^{p}\left[\xi_{11}^{T}(i, q) Q \xi_{11}(i, q)-\xi_{10}^{T}(i, 0) Q \xi_{10}(i, 0)\right] \\
& +\sum_{j=0}^{q}\left[\sum_{\kappa=-d_{1}}^{-1} \xi_{\kappa 1}^{T}(p+1, j) Q_{1} \xi_{\kappa 1}(p+1, j)-\sum_{\kappa=-d_{1}}^{-1} \xi_{\kappa 1}^{T}(0, j) Q_{1} \xi_{\kappa 1}(0, j)\right] \\
& +\sum_{i=0}^{p}\left[\sum_{\kappa=-d_{2}}^{-1} \xi_{1 \kappa}^{T}(i, q+1) Q_{2} \xi_{1 \kappa}(i, q+1)-\sum_{\kappa=-d_{2}}^{-1} \xi_{1 \kappa}^{T}(i, 0) Q_{2} \xi_{1 \kappa}(i, 0)\right]
\end{aligned}
$$

Thus, together with (40) implies that

$$
\begin{aligned}
\|\bar{e}(i, j)\|_{2}^{2}-\gamma^{2}\|\bar{\omega}(i, j)\|_{2}^{2}+\|\mu(i, j)\|_{2}^{2}< & \sum_{j=0}^{\infty} \xi_{01}^{T}(0, j)(P-Q) \xi_{01}(0, j)+\sum_{i=0}^{\infty} \xi_{10}^{T}(i, 0) Q \xi_{10}(i, 0) \\
& +\sum_{j=0}^{\infty} \sum_{\kappa=-d_{1}}^{-1} \xi_{\kappa 1}^{T}(0, j) Q_{1} \xi_{\kappa 1}(0, j)+\sum_{i=0}^{\infty} \sum_{\kappa=-d_{2}}^{-1} \xi_{1 \kappa}^{T}(i, 0) Q_{2} \xi_{1 \kappa}(i, 0)
\end{aligned}
$$

which implies that

$$
\begin{aligned}
\|\bar{e}(i, j)\|_{2}^{2}+\|\mu(i, j)\|_{2}^{2}< & \gamma^{2}\left[\|\bar{\omega}(i, j)\|_{2}^{2}+\sum_{j=0}^{\infty} \xi_{01}^{T}(0, j) \tilde{P} \xi_{01}(0, j)+\sum_{i=0}^{\infty} \xi_{10}^{T}(i, 0) \tilde{Q} \xi_{10}(i, 0)\right. \\
& \left.+\sum_{j=0}^{\infty} \sum_{\kappa=-d_{1}}^{-1} \xi_{\kappa 1}^{T}(0, j) \tilde{Q}_{1} \xi_{\kappa 1}(0, j)+\sum_{i=0}^{\infty} \sum_{\kappa=-d_{2}}^{-1} \xi_{1 \kappa}^{T}(i, 0) \tilde{Q}_{2} \xi_{1 \kappa}(i, 0)\right]
\end{aligned}
$$


where $P-Q<\gamma^{2} \tilde{P}, Q<\gamma^{2} \tilde{Q}, Q_{1}<\gamma^{2} \tilde{Q}_{1}$ and $Q_{2}<\gamma^{2} \tilde{Q}_{2}$. Now, to establish the $\mathcal{H}_{\infty}$ performance, we show that there exists a scalar $\alpha>0$ such that

$$
\begin{aligned}
\|\mu(i, j)\|_{2}^{2} \geq & \alpha^{2}\left[\|\bar{\omega}(i, j)\|_{2}^{2}+\sum_{j=0}^{\infty} \xi_{01}^{T}(0, j) \tilde{P} \xi_{01}(0, j)+\sum_{i=0}^{\infty} \xi_{10}^{T}(i, 0) \tilde{Q} \xi_{10}(i, 0)\right. \\
& \left.+\sum_{j=0}^{\infty} \sum_{\kappa=-d_{1}}^{-1} \xi_{\kappa 1}^{T}(0, j) \tilde{Q}_{1} \xi_{\kappa 1}(0, j)+\sum_{i=0}^{\infty} \sum_{\kappa=-d_{2}}^{-1} \xi_{1 \kappa}^{T}(i, 0) \tilde{Q}_{2} \xi_{1 \kappa}(i, 0)\right]
\end{aligned}
$$

Consider the inverse system of (5):

$$
\begin{aligned}
\xi(i+1, j+1)= & \tilde{A}_{1} \xi(i, j+1)+\tilde{A}_{2} \xi(i+1, j)+\tilde{A}_{d 1} \xi\left(i-d_{1}, j+1\right)+\tilde{A}_{d 2} \xi\left(i+1, j-d_{2}\right) \\
& +\tilde{B}_{1} \omega(i, j+1)+\tilde{B}_{2} \omega(i+1, j) \\
\triangleq & \mathcal{A} \eta(i, j)+\mathcal{B} \bar{\omega}(i, j) \\
= & \left(\mathcal{A}+\mathcal{B} \Phi^{-1} \Omega^{T}\right) \eta(i, j)+\mathcal{B} \Phi^{-\frac{1}{2}} \mu(i, j) \\
\bar{\omega}(i, j)= & \Phi^{-1} \Omega^{T} \eta(i, j)+\Phi^{-\frac{1}{2}} \mu(i, j)
\end{aligned}
$$

where $\mathcal{A} \triangleq\left[\begin{array}{cccc}\tilde{A}_{1} & \tilde{A}_{2} & \tilde{A}_{d 1} & \tilde{A}_{d 2}\end{array}\right], \mathcal{B} \triangleq\left[\begin{array}{cc}\tilde{B}_{1} & \tilde{B}_{2}\end{array}\right]$ and $\eta(i, j)$ has been defined before. It can be verified from (9) that the system in (45) is asymptotically stable, thus there exists a bounded $\beta>0$ such that

$$
\begin{aligned}
& \|\bar{\omega}(i, j)\|_{2}^{2}+\left[\sum_{j=0}^{\infty} \xi_{01}^{T}(0, j)(\tilde{P}-\tilde{Q}) \xi_{01}(0, j)+\sum_{i=0}^{\infty} \xi_{10}^{T}(i, 0) \tilde{Q} \xi_{10}(i, 0)\right. \\
& \left.\quad+\sum_{j=0}^{\infty} \sum_{\kappa=-d_{1}}^{-1} \xi_{\kappa 1}^{T}(0, j) \tilde{Q}_{1} \xi_{\kappa 1}(0, j)+\sum_{i=0}^{\infty} \sum_{\kappa=-d_{2}}^{-1} \xi_{1 \kappa}^{T}(i, 0) \tilde{Q}_{2} \xi_{1 \kappa}(i, 0)\right] \leq \beta^{2}\|\mu(i, j)\|_{2}^{2}
\end{aligned}
$$

This implies (44) with $\beta=\frac{1}{\alpha}$. With zero boundary conditions as in (2), we can easily obtain (8), hence the proof is completed.

\section{REFERENCES}

[1] W. Assawinchaichote and S. K. Nguang, $H_{\infty}$ filtering for nonlinear singularly perturbed systems with pole placement constraints: an LMI Approach, IEEE Trans. Signal Processing, 34(6):1659-1667, Jun. 2004.

[2] S. Boyd, L. El Ghaoui, E. Feron and V. Balakrishnan. Linear Matrix Inequalities in Systems and Control Theory. SIAM, Philadelphia, PA, 1994.

[3] C. E. de Souza and A. Trofino. An LMI approach to the design of robust $H_{2}$ filters. In Recent Advances on Linear Matrix Inequality Methods in Control, Philadelphia, PA, 1999.

[4] C. Du, L. Xie, and Y. C. Soh. $H_{\infty}$ fltering of 2-D discrete systems. IEEE Trans. Signal Processing, 48(6):1760-1768, 2000.

[5] C. Du, L. Xie and C. Zhang, $H_{\infty}$ control and robust stabilization of two-dimensional systems in Roesser models, Automatica, $37: 205-211,2001$.

[6] E. Fornasini and G. Marchesini. Doubly indexed dynamical systems: state-space models and structual properties. Math. Syst. Theory, 12:59-72, 1978.

[7] H. Gao, J. Lam, C. Wang, and S. Xu. $H_{\infty}$ model reduction for uncertain two-dimensional discrete systems. Optim. Control Appl. Meth., 26:199-227, 2005.

[8] H. Gao, J. Lam, S. Xu, and C. Wang. Stabilization and $H_{\infty}$ control of two-dimensional Markovian jump systems. IMA J. Math. and Control Info., 21:377-392, 2004.

[9] H. Gao and C. Wang. Delay-dependent robust $H_{\infty}$ and $L_{2}-L_{\infty}$ filtering for a class of uncertain nonlinear time-delay systems. IEEE Trans. Automat. Control, 48(9):1661-1666, 2003.

[10] J. C. Geromel and M. C. De Oliveira. $H_{2}$ and $H_{\infty}$ robust filtering for convex bounded uncertain systems. IEEE Trans. Automat. Control, 46(1):100-107, 2001.

[11] E. Gershon, D. J. N. Limebeer, U. Shaked, and I. Yaesh. Robust $H_{\infty}$ fltering of stationary continuoustime linear systems with stochastic uncertainties. IEEE Trans. Automat. Control, 46(11):1788-1793, 2001. 
[12] T. Hinamoto. 2-D Lyapunov equation and filter design based on Fornasini-Marchesini second model. IEEE Trans. Circuits and Systems: Part I, 40(2):102-110, 1993.

[13] T. Kaczorek. Two-Dimensional Linear Systems. Springer-Verlag, Berlin, Germany, 1985.

[14] H. Liu, F. Sun, K. He and Z. Sun, Design of reduced-order $H_{\infty}$ filter for Markovian jumping systems with time delay, IEEE Trans. Circuits and Systems -II, 51(11):607-612, Nov. 2004.

[15] W. S. Lu and A. Antoniou. Two-Dimensional Digital Filters. Marcel Dekker, New York, 1992.

[16] Marszalek, W. Two-dimensional state-space discrete models for hyperbolic partial differential equations. Applied Mathematical Modeling, 8:11-14, 1984.

[17] A. Megretski and A. Rantzer, System analysis via integral quadratic constraints. IEEE Trans. Automatic Control, 42(6): 819-830, 1997.

[18] R. M. Palhares, C. E. de Souza, and P. L. D. Peres. Robust $H_{\infty}$ filtering for uncertain discrete-time state-delayed systems. IEEE Trans. Signal Processing, 49(8):1696-1703, 2001.

[19] W. Paszke, J. Lam, K. Galkowski, S. Xu, and Z. Lin. Robust stability and stabilisation of 2D discrete state-delayed systems. Systems \& Control Letters, 51(3-4):277-291, 2004.

[20] H. D. Tuan, P. Apkarian, and T. Q. Nguyen. Robust mixed $H_{2} / H_{\infty}$ filtering of 2-D systems. IEEE Trans. Signal Processing, 50(7):1759-1771, 2002.

[21] Z. Wang, B. Huang, and H. Unbehauen. Robust $H_{\infty}$ observer design of linear state delayed systems with parametric uncertainty: the discrete-time case. Automatica, 35:1161-1167, 1999.

[22] Z. Wang, F. Yang, D. W. C. Ho and X. Liu, Robust $H_{\infty}$ filtering for stochastic time-delay systems with missing measurements, IEEE Trans. Signal Processing, 54(7):2579-2587, 2006.

[23] Z. Wang, F. Yang and X. Liu, Robust filtering for systems with stochastic nonlinearities and deterministic uncertainties, Proceedings of IMechE - Journal of Systems and Control Engineering, 220(3):171-182, 2006.

[24] Z. Wang, F. Yang, D. W. C. Ho and X. Liu, Robust finite-horizon filtering for stochastic systems with missing measurements, IEEE Signal Processing Letters, 12(6):437-440, 2005.

[25] L. Wu, P. Shi, C. Wang, and H. Gao. Delay-dependent robust $H_{\infty}$ and $L_{2}-L_{\infty}$ filtering for LPV systems with both discrete and distributed delays. IEE Proc. Part D: Control Theory Appl., 153(4):483-492, 2006.

[26] L. Xie, C. Du, Y. C. Soh, and C. Zhang. $H_{\infty}$ and robust control of 2-D systems in FM second model. Multidimensional Systems and Signal Processing, 13:256-287, 2002.

[27] S. Xu and P. V. Dooren. Robust $H_{\infty}$ filtering for a class of nonlinear systems with state delay and parameter uncertainty. Int. J. Control, 75(10):766-774, 2002.

[28] D. Yue and Q.-L. Han, Robust $H_{\infty}$ filter design of uncertain descriptor systems with discrete and distributed delays, IEEE Trans. Signal Processing, 52(11):3200-3212, Nov. 2004.

[29] H. Zhang, D. Zhang and L. Xie, An innovation approach to $H_{\infty}$ prediction with application to systems with delayed measurements, Automatica, 40(7):1253-1261, 2004. 


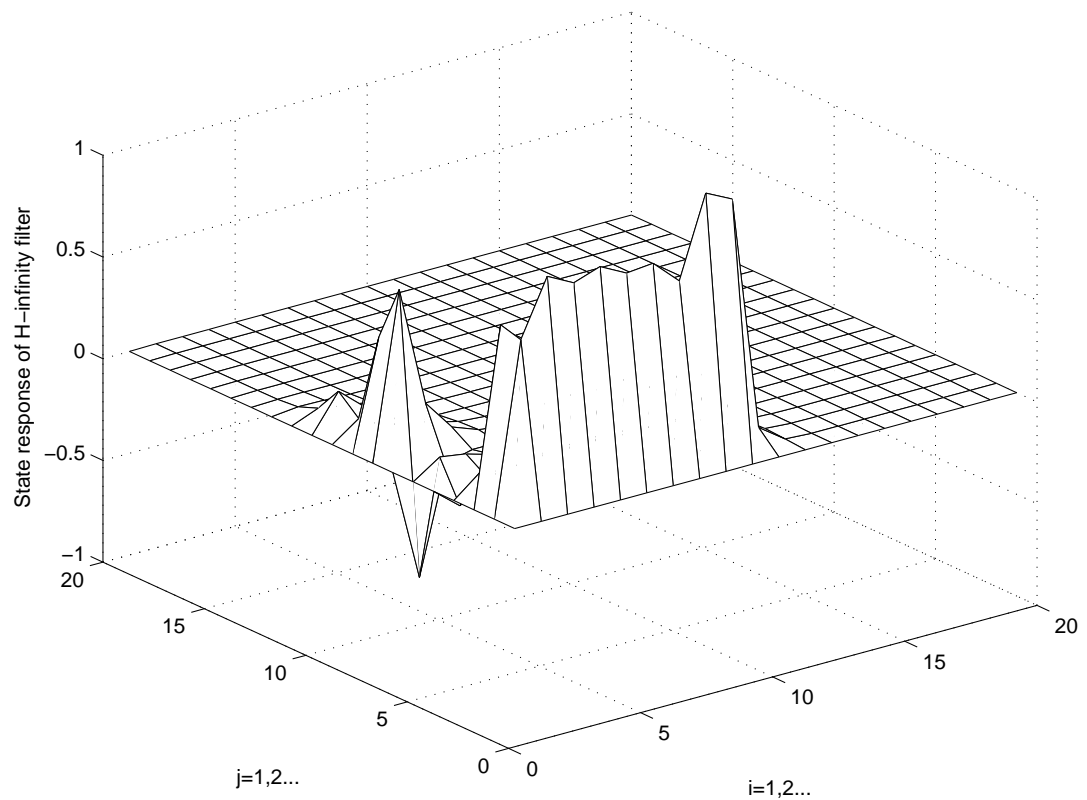

Fig. 1. State response of the $\mathcal{H}_{\infty}$ filter $x_{f 1}(i, j)$

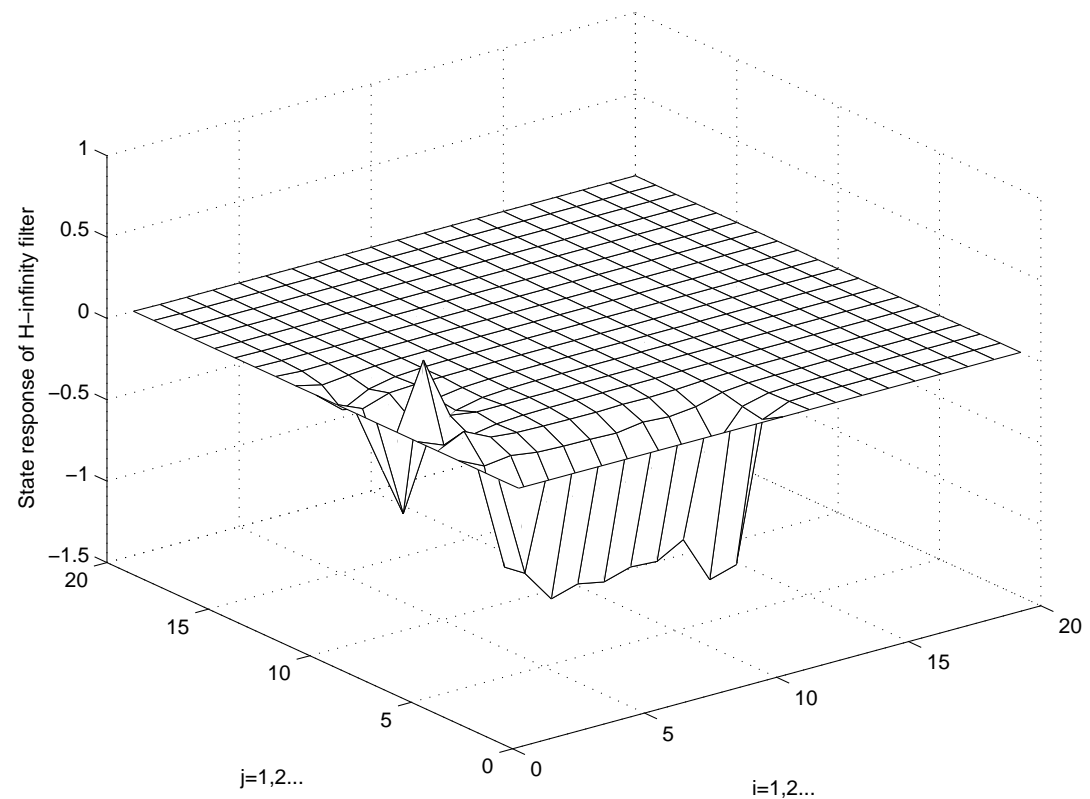

Fig. 2. State response of the $\mathcal{H}_{\infty}$ filter $x_{f 2}(i, j)$ 


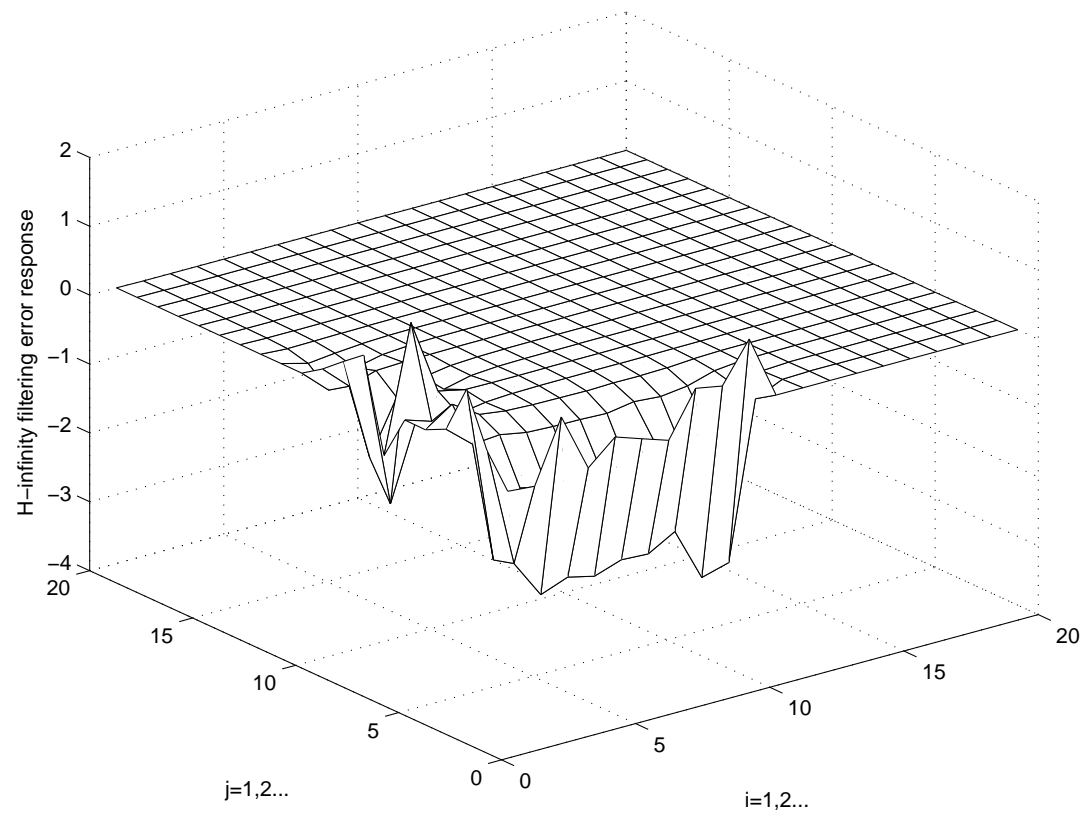

Fig. 3. Error response $e_{1}(i, j)$

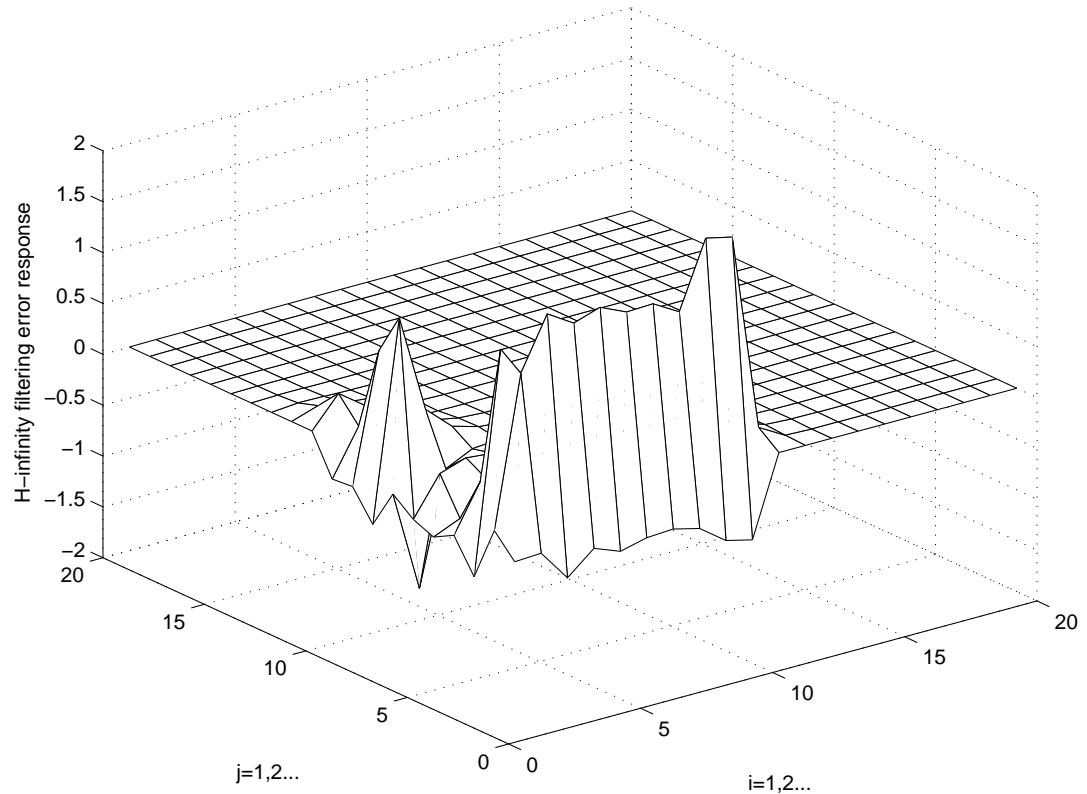

Fig. 4. Error response $e_{2}(i, j)$ 